\title{
Neuropathology of Cerebral Ischemia
}

\section{"Masoud Fereidoni', Bahram Farhadi Moghadam", Arash Abdolmaleki²,3}

${ }^{1}$ Department of Biology, Faculty of Science, Ferdowsi University of Mashhad, Mashhad, Iran

${ }^{2}$ Department of Engineering Sciences, Faculty of Advanced Technologies, University of Mohaghegh Ardabili, Namin, Iran ${ }^{3}$ Bio Science and Biotechnology Research Center (BBRC), Sabalan University of Advanced Technologies (SUAT), Namin, Iran

\section{Article Info:}

\section{ABSTRACT}

Introduction: Cerebral ischemia results from glucose and oxygen reduction following insufficiency of brain blood supply. Ischemia could be induced in focal and diffuse models. A type of animal model of transient brain global ischemia is induced via common carotid arteries ligation and focal ischemia is induced by middle cerebral artery obstructions, which are the most common approach for investigation of the pathophysiology of brain ischemia and its mechanisms. Understanding of pathophysiological mechanisms of cerebral ischemia is important for the development of novel preventive and therapeutic approaches for brain ischemia. Conclusion: In the present study, we have described the pathophysiological mechanisms of brain ischemic events, such as decreases in cerebral blood flow, glutamate neurotoxicity, oxidative stress, inflammation, brain edema, cholinergic system dysfunction, neural cell death, and hippocampal damage. Extensive efforts are being performed to find effective drugs with the least side effects for the modulation of pathways involved in brain ischemia. In summation, represented information may be an appropriate guide to design novel therapeutic strategies for brain ischemia.

*Corresponding Author: Arash Abdolmaleki

Email: Abdolmalekiarash1364@gmail.com

Keywords:

1. Neuropathology

2. Stroke

3. Brain Ischemia

4. Nervous System Diseases 


\section{نوروياتولوزى ايسكمى مغزى}

\section{مسعود فريدونى'، بجهرام فرهادى مقدم'، آرش عبدالملكى "r."}

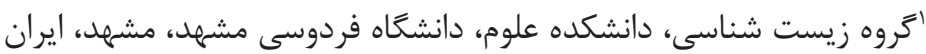

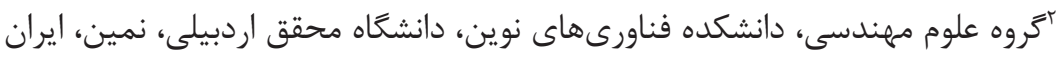

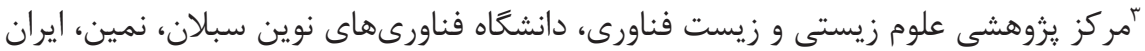

\section{اطلاعات مقاله:}

يذيرش: r بهمن و وسا

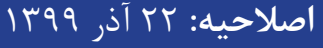

دريافت: ل ه مهر وج"

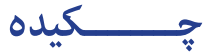

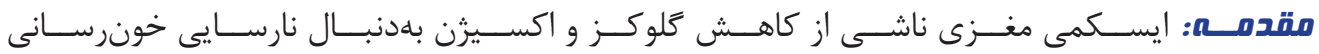

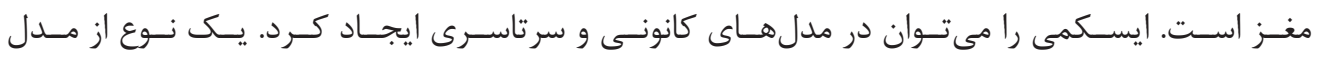

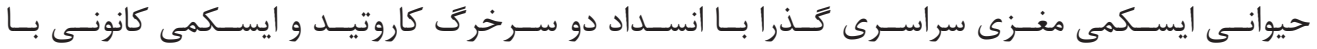

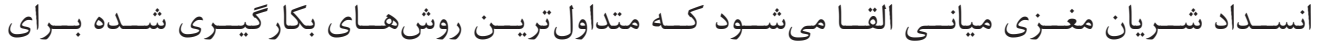

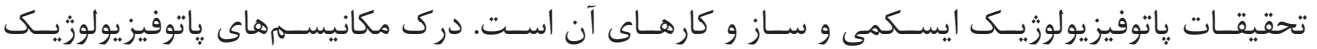

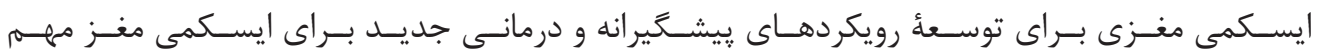

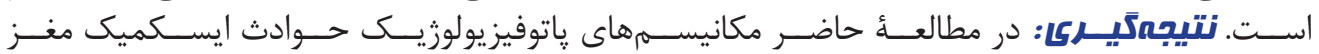

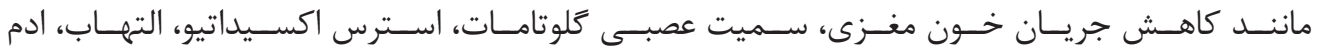

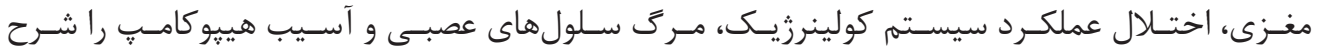

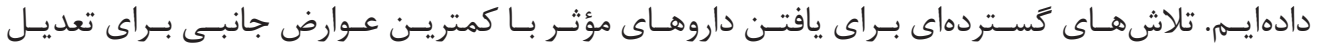

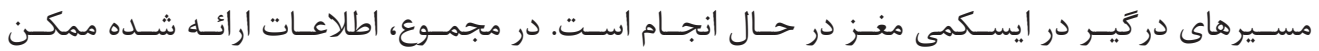

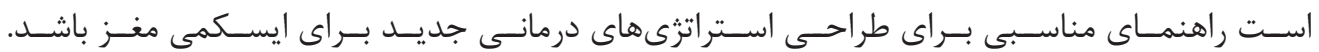

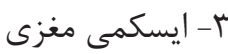

ا

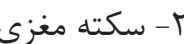

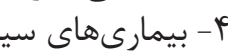

$$
\text { *ويسنده مسئول: آرش عبدالملكى }
$$




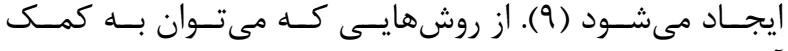

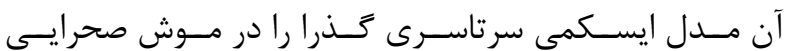

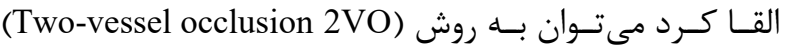

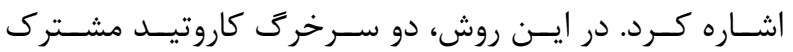

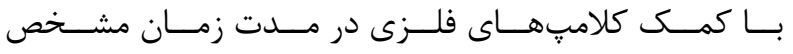

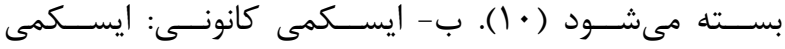

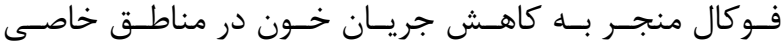

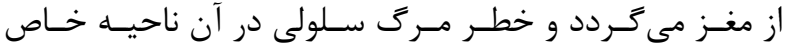

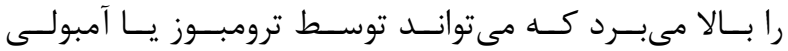

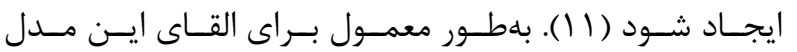

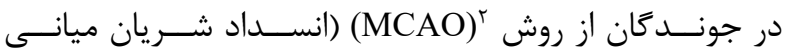

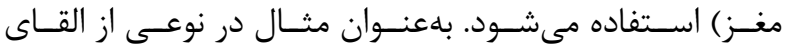

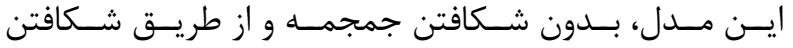

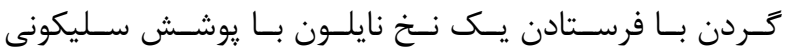

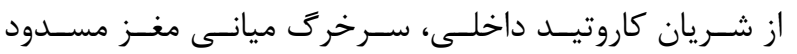

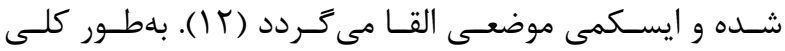

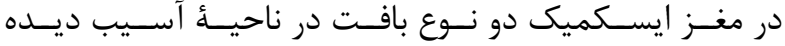

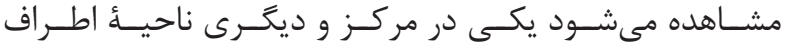

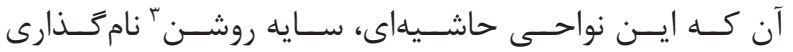

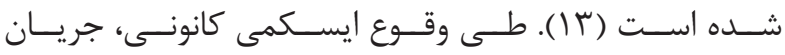

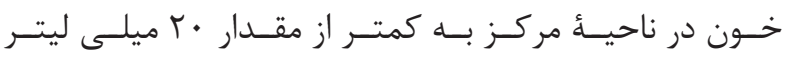

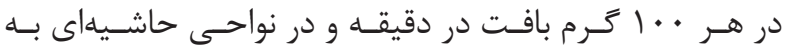

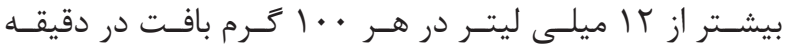

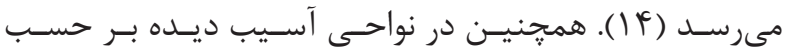

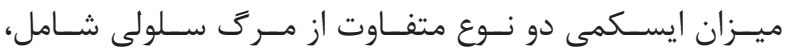

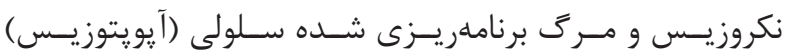

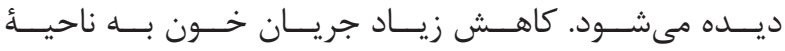

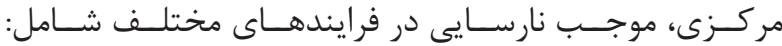

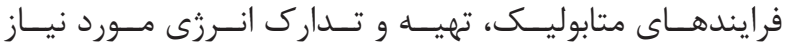

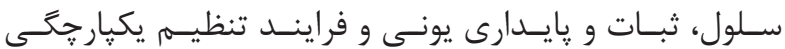

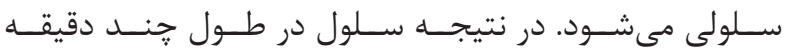

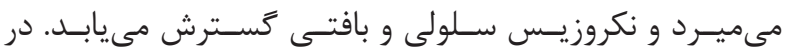

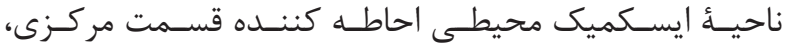

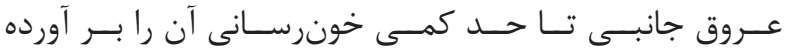

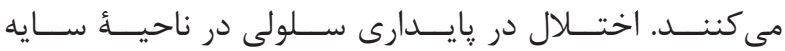

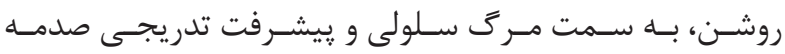

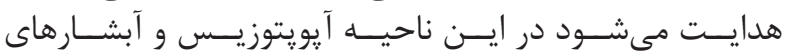

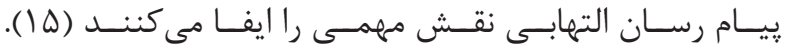

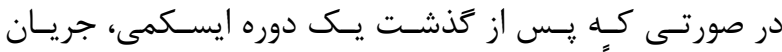

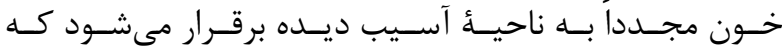

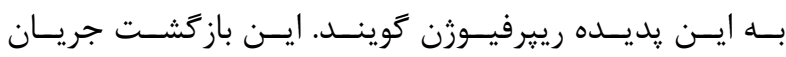

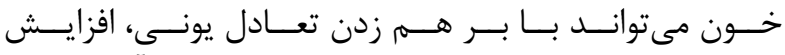

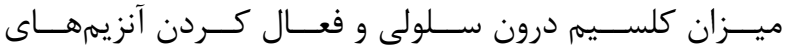

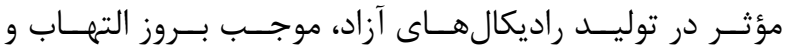

${ }^{1}$ Plateau

${ }^{2}$ Middle Cerebral Artery Occlusion

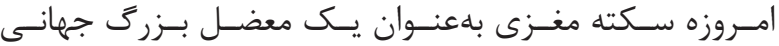

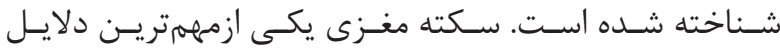

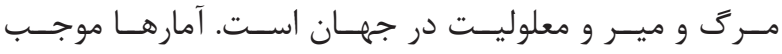

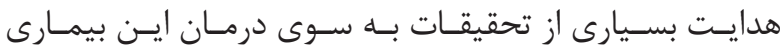

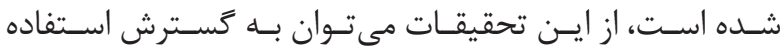

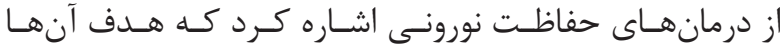

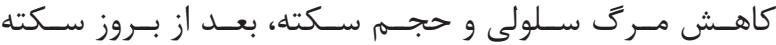

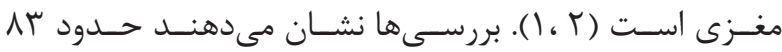

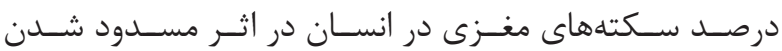

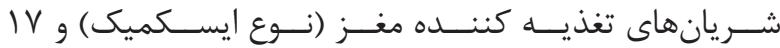

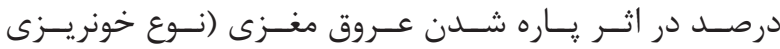

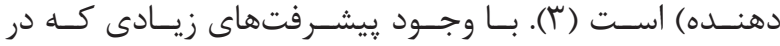

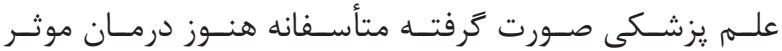

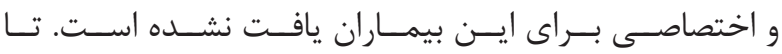

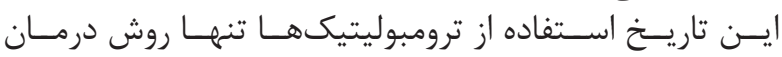

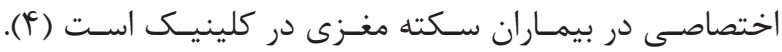

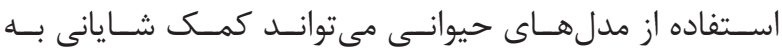

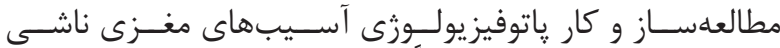

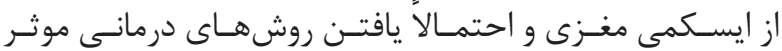

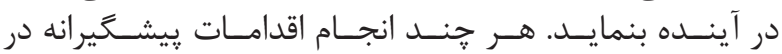

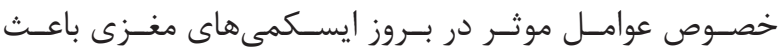

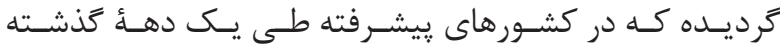

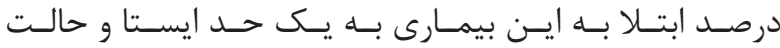

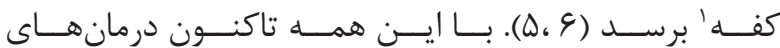

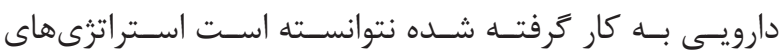

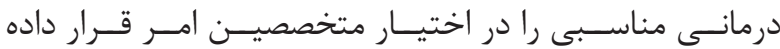

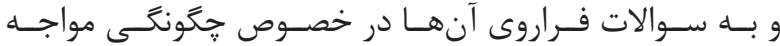

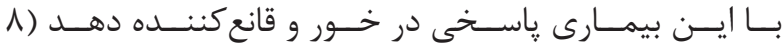

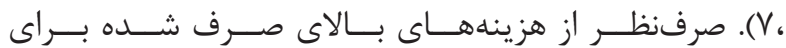

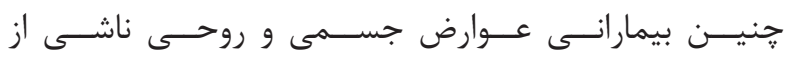

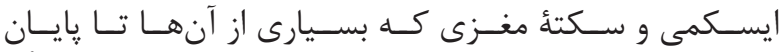

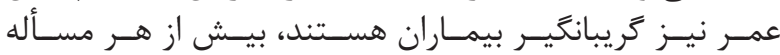

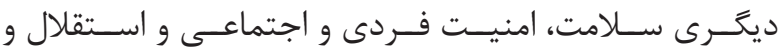

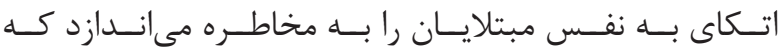

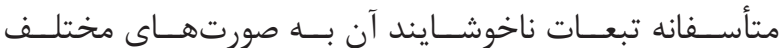

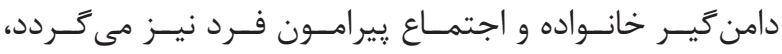

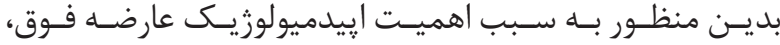

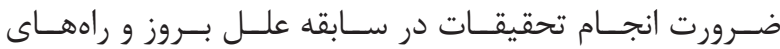

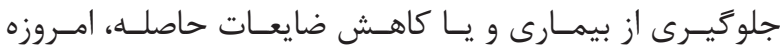

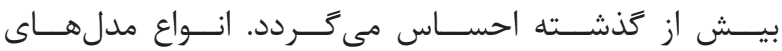

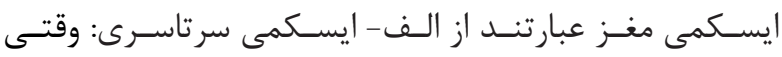

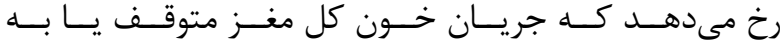

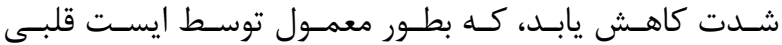




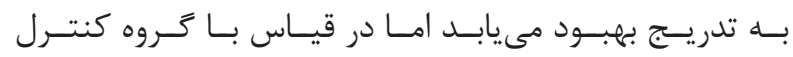

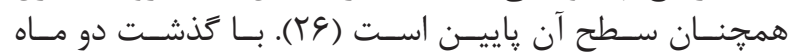

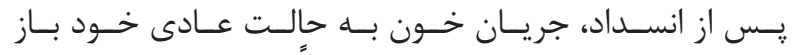

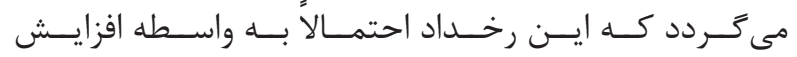

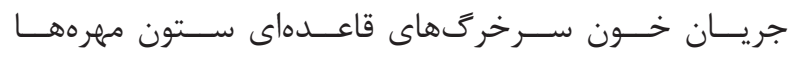

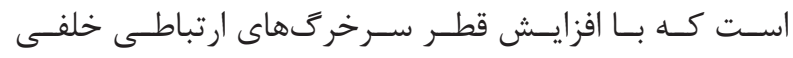

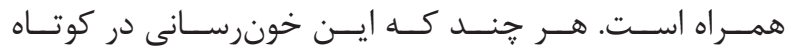

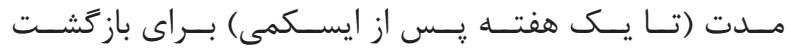

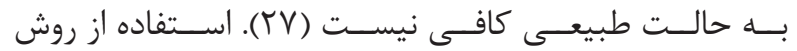

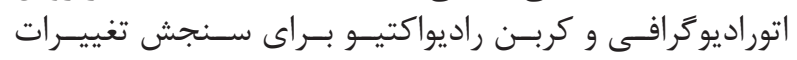

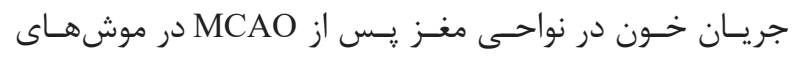

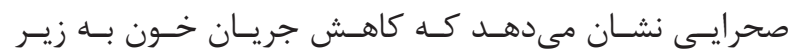

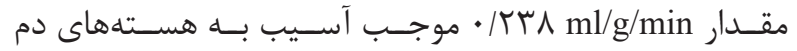

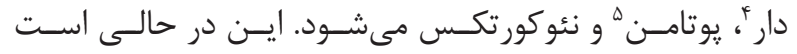

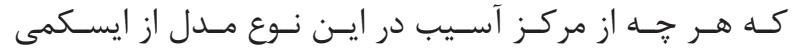

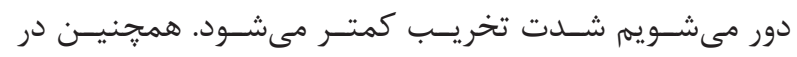

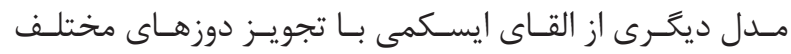

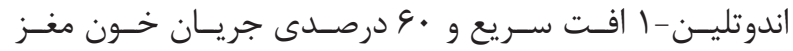

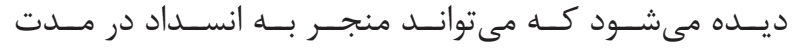

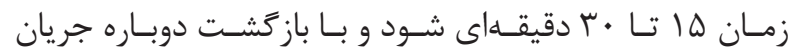

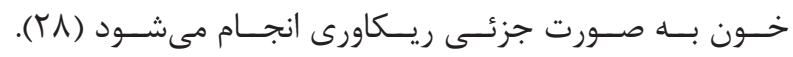

\section{سـميت تحريكى ناشسى از اختلال در سيستم كلوتاماترزيك}

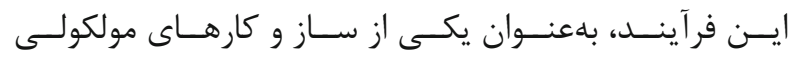

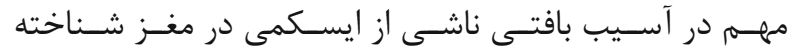

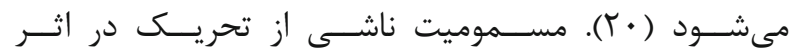

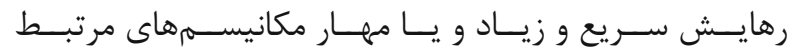

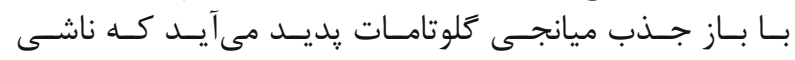

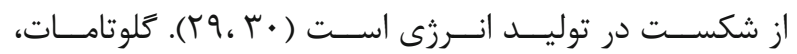

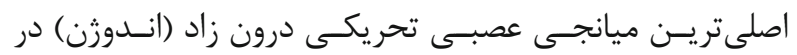

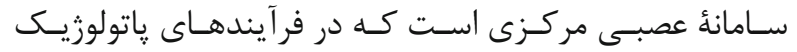

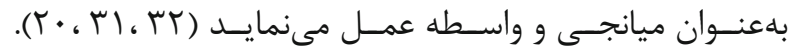

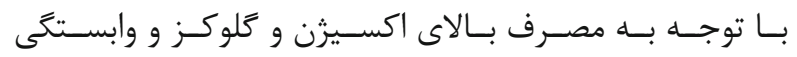

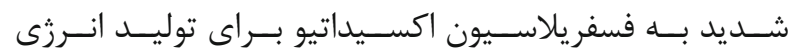

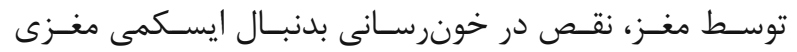

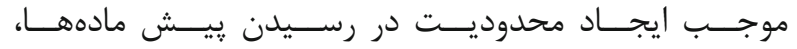

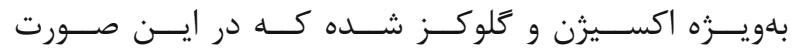

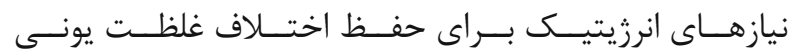

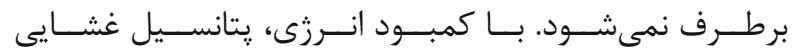

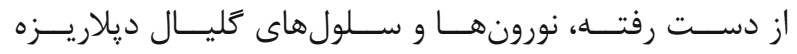

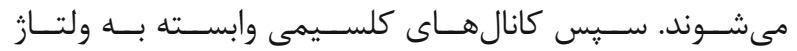

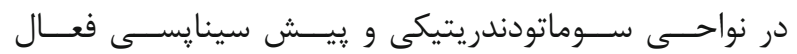

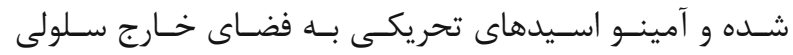

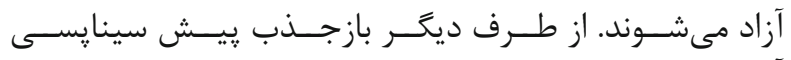

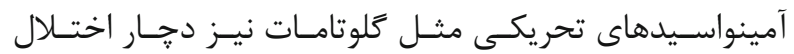

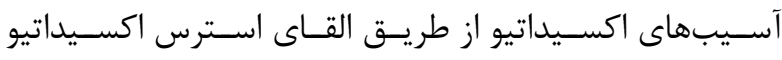

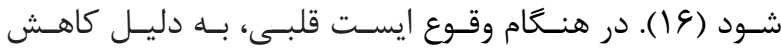

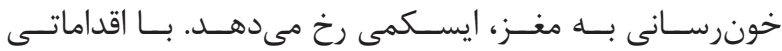

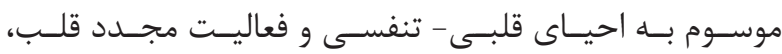

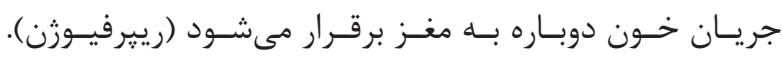

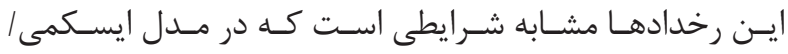

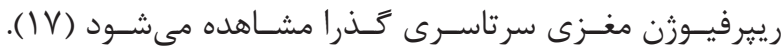

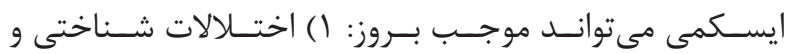

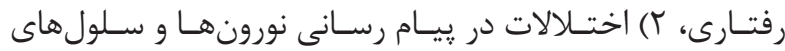

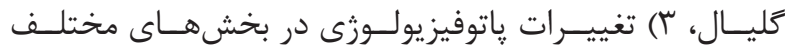

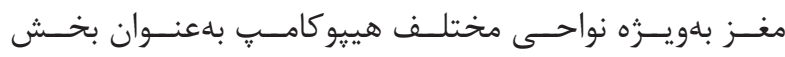

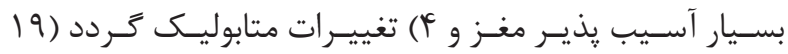

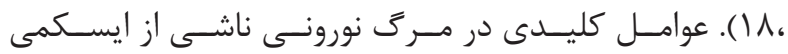

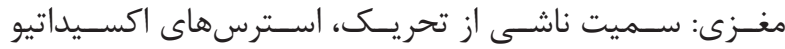

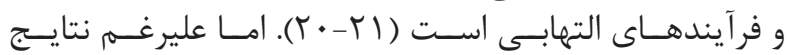

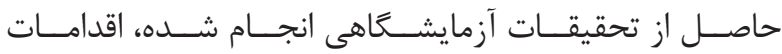

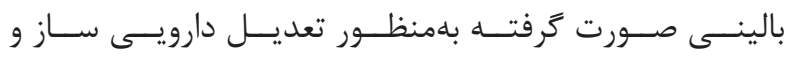

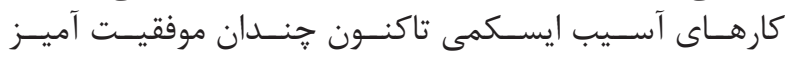

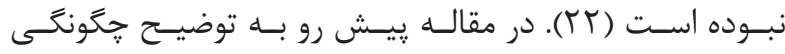

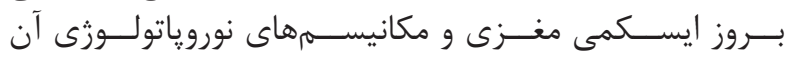

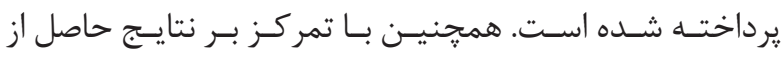

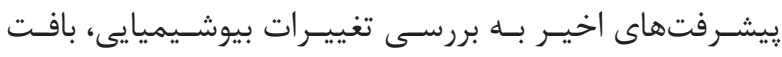

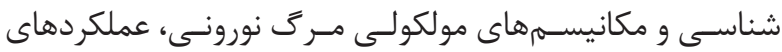

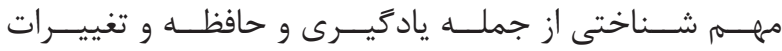

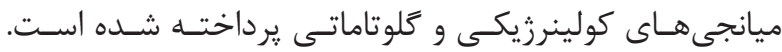

\section{اختلال در جريان خونر سانى به مغز}

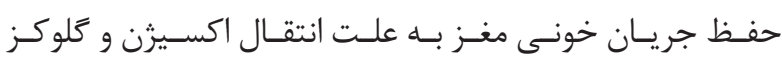

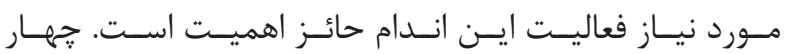

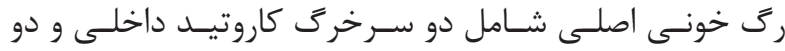

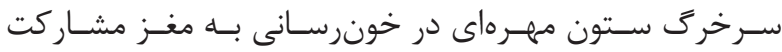

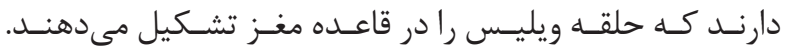

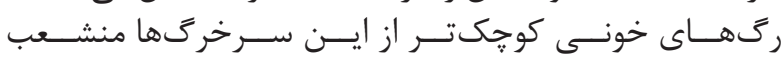

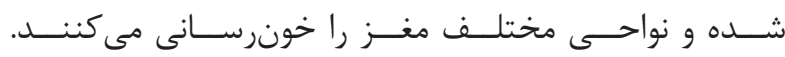

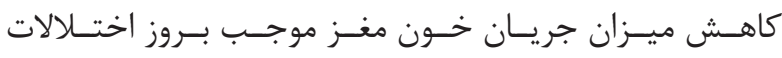

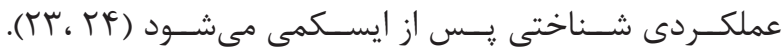

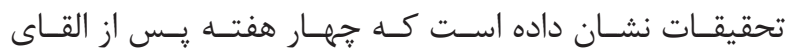

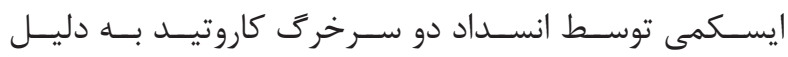

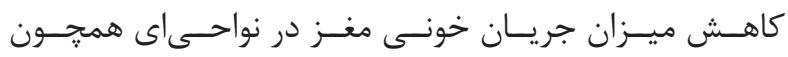

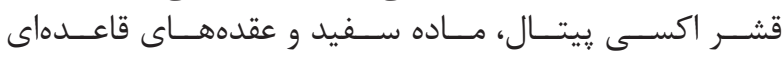

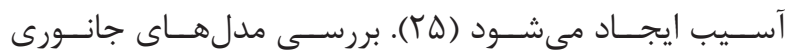

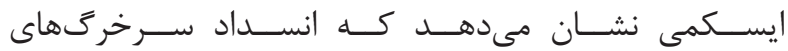

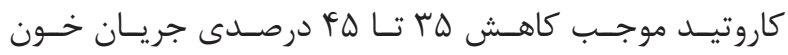

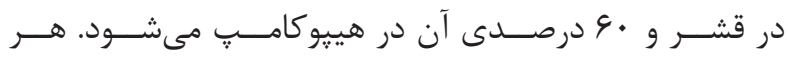

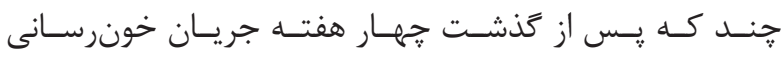




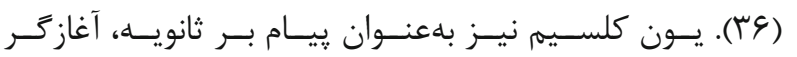

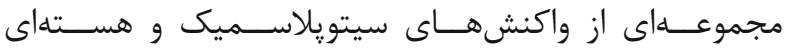

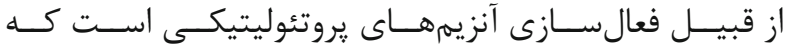

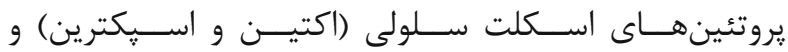

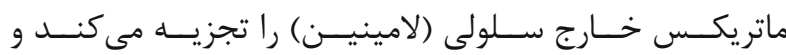

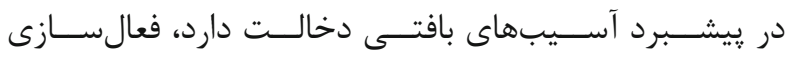

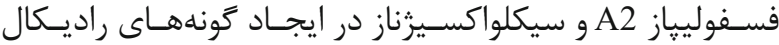

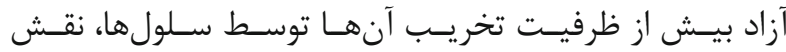

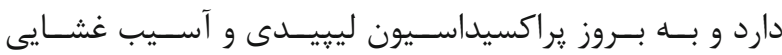

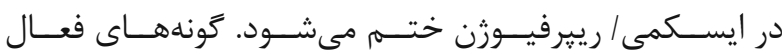

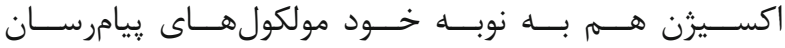

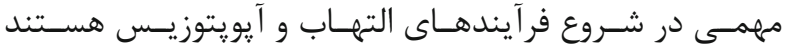

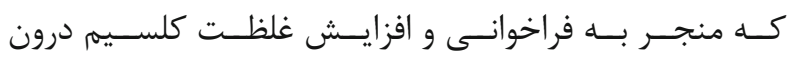

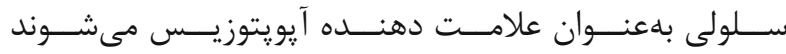

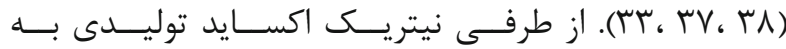

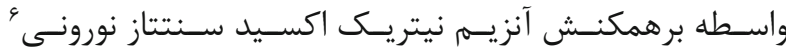

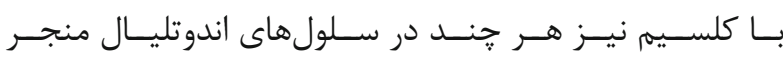

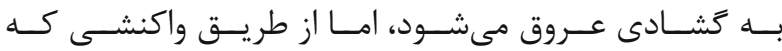

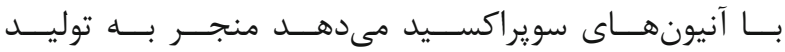

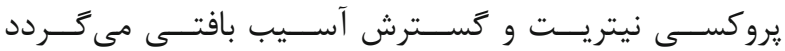

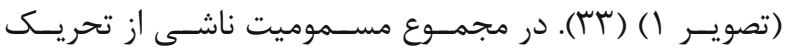

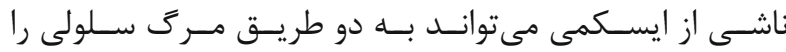

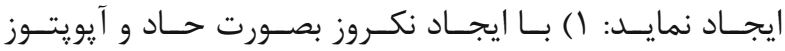

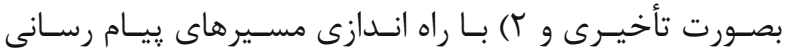

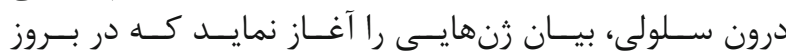

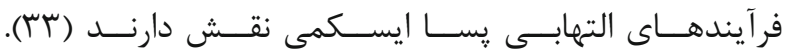

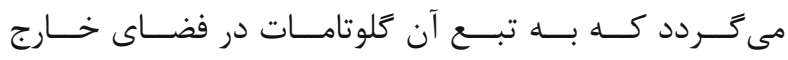

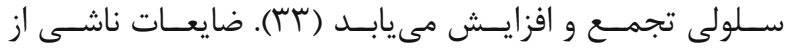

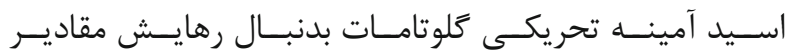

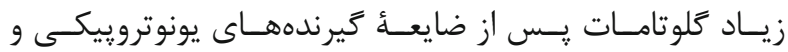

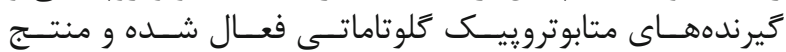

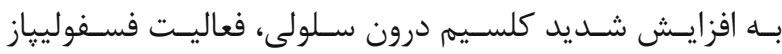

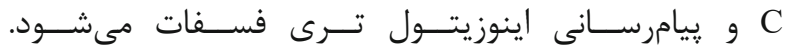

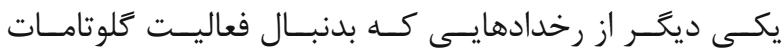

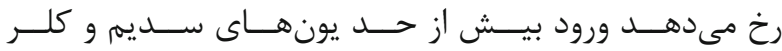

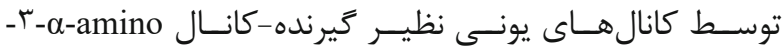
isoxazolepropionic acid (AMPA)- - -methyl-0-hydroxy

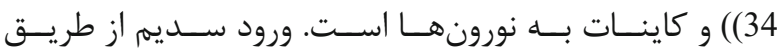

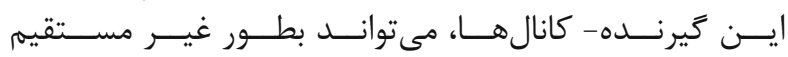

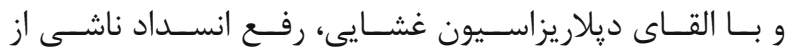

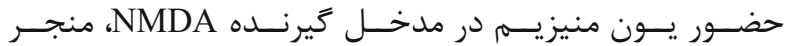

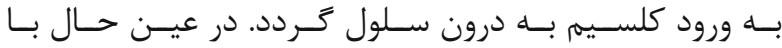

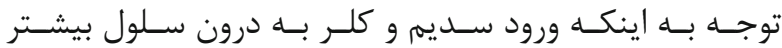

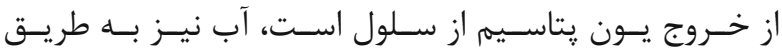

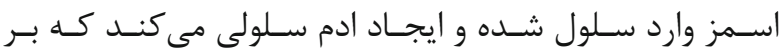

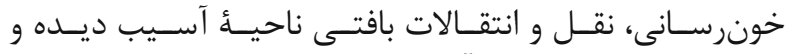

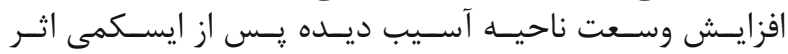

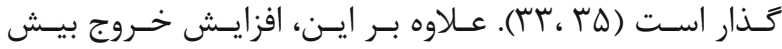

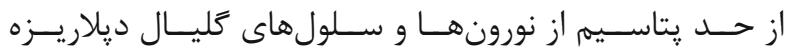

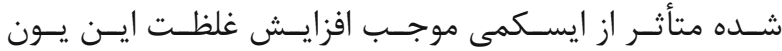

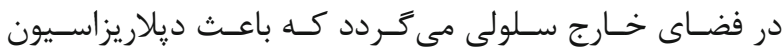

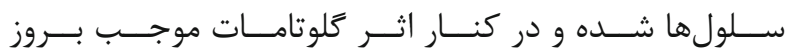

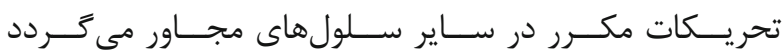

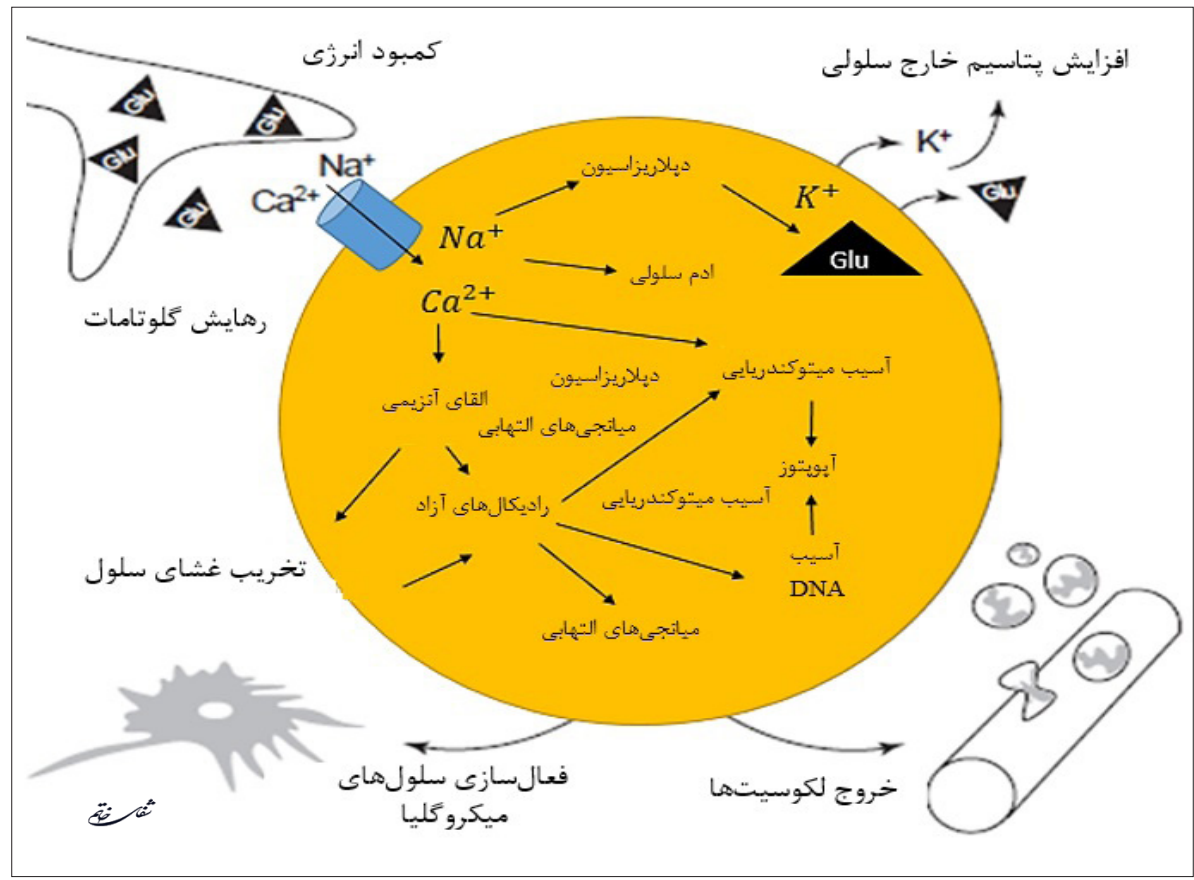

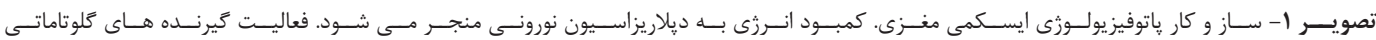

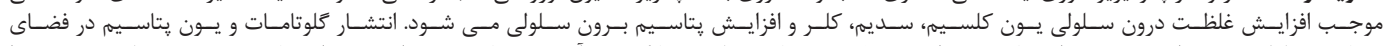

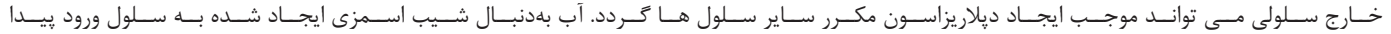

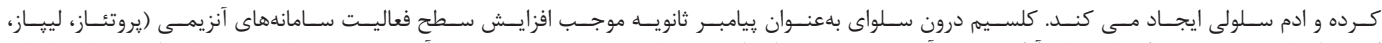

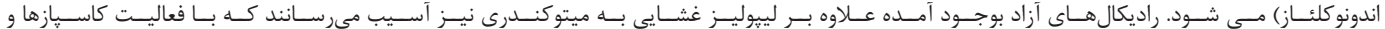

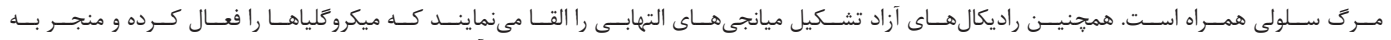

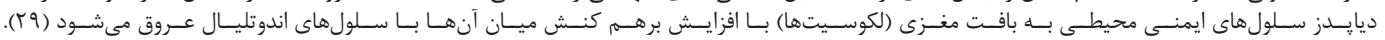




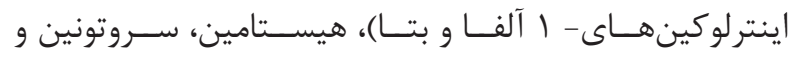

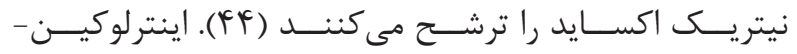

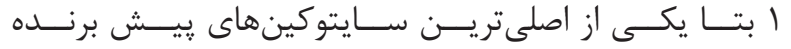

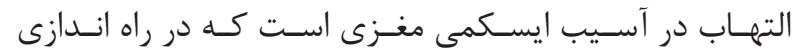

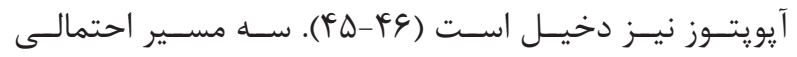

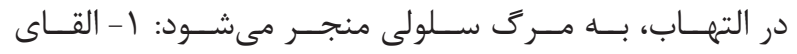

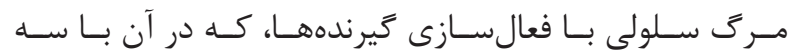

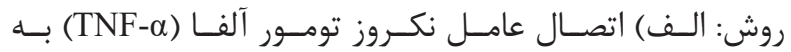

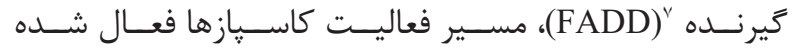

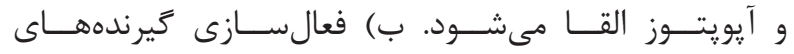

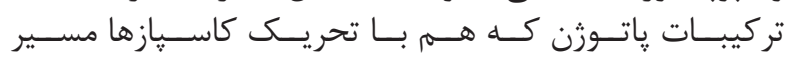

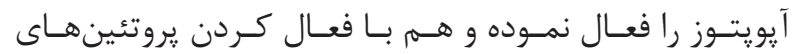

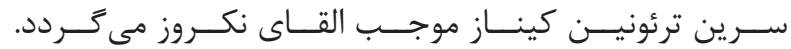

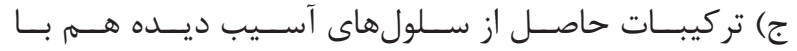

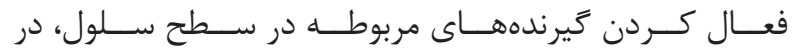

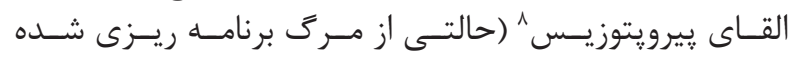

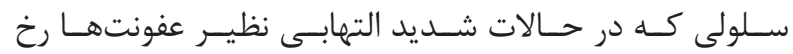

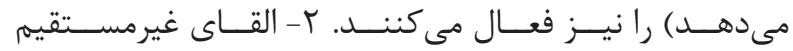

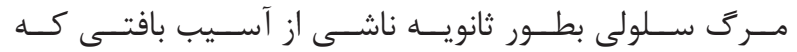

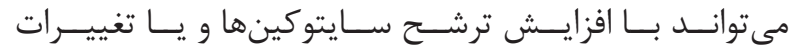

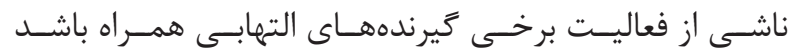

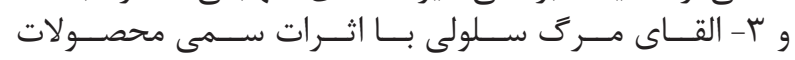

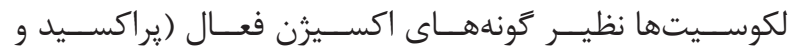

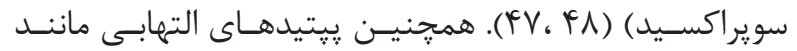

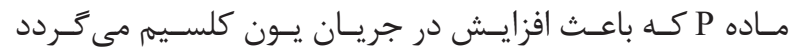

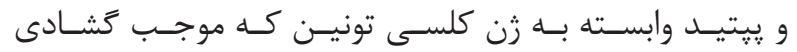

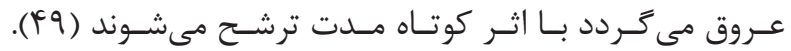

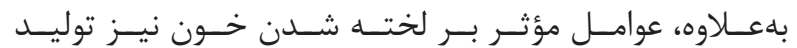

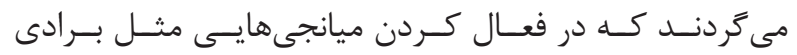

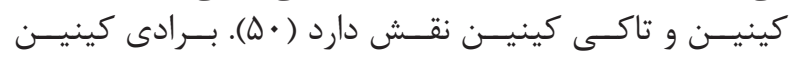

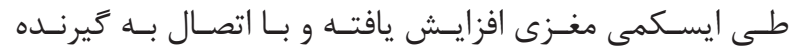

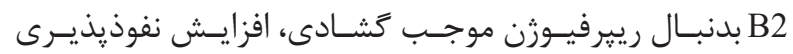

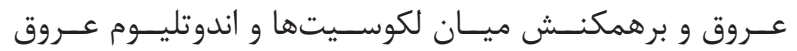

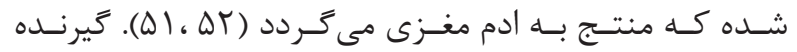

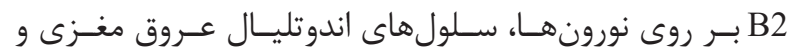

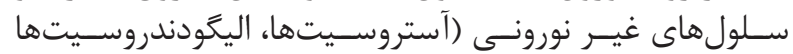

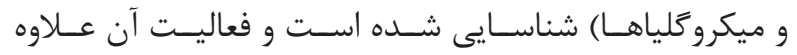

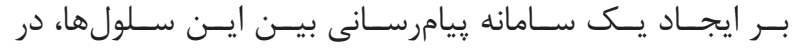

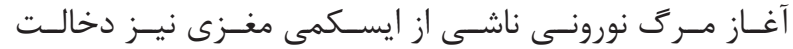

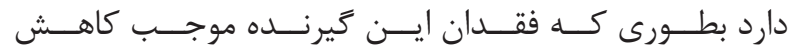

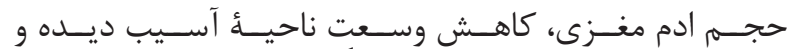

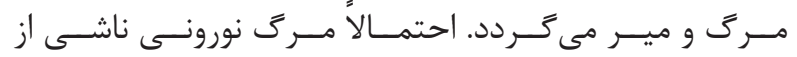

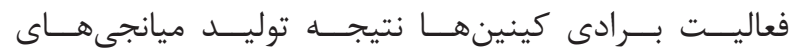

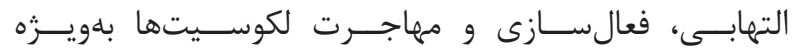

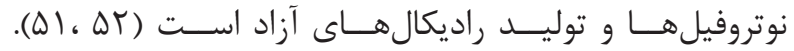

\section{توليد و فعاليت راديكالهاى آزاد}

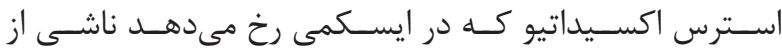

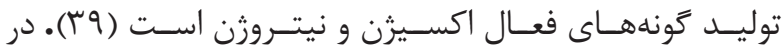

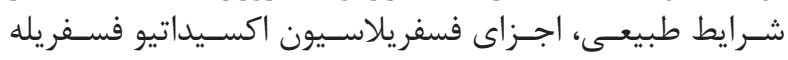

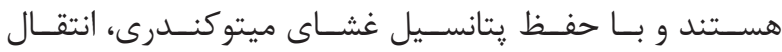

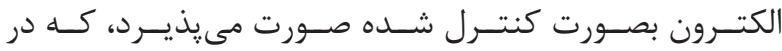

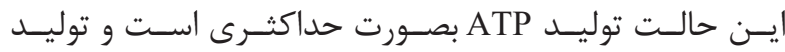

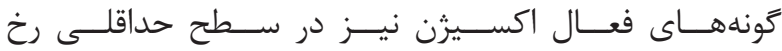

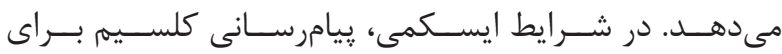

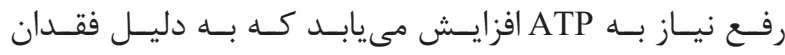

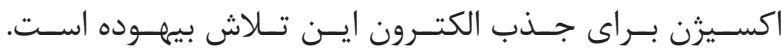

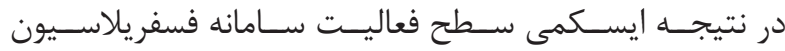

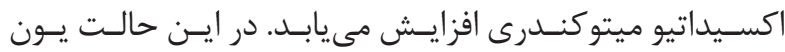

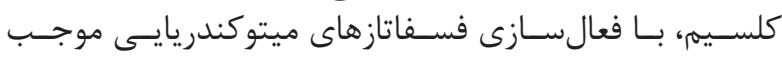

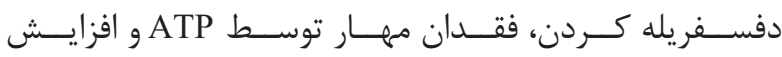

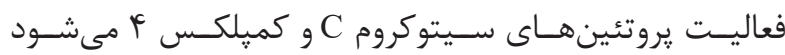

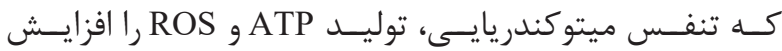

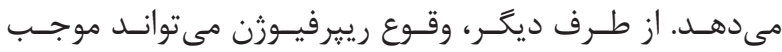

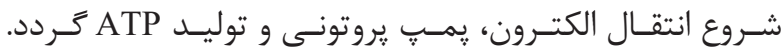

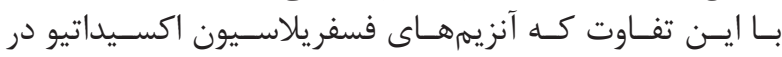

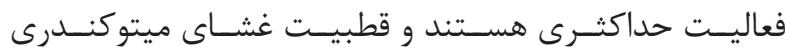

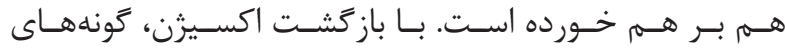

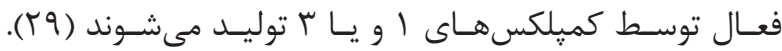

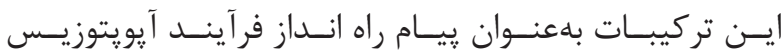

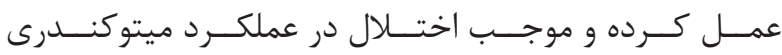

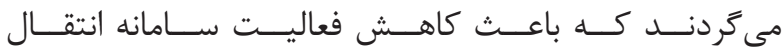

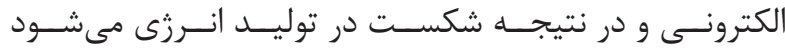

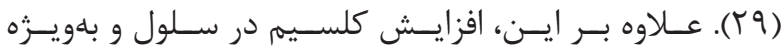

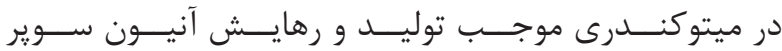

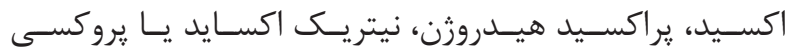

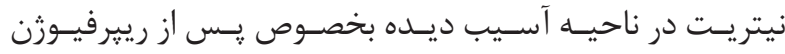

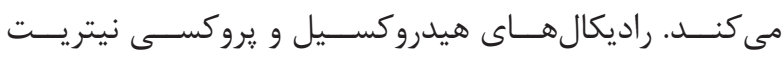

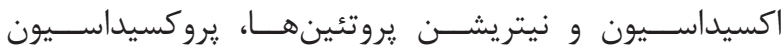

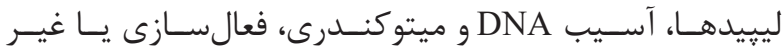

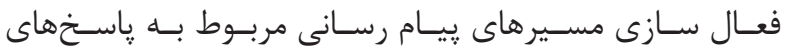

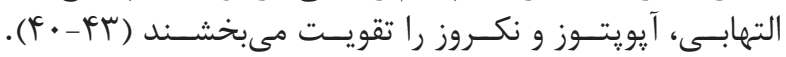
التهاب

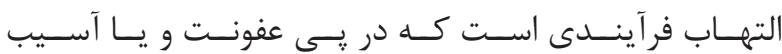

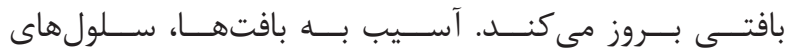

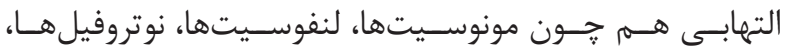

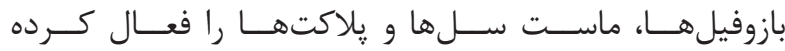

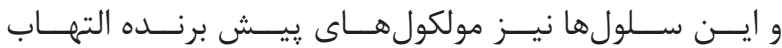

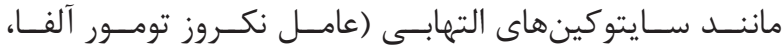

${ }^{7}$ FAS-Associated Via Death Domain

${ }^{8}$ Pyro Ptosis 


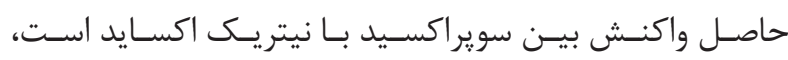

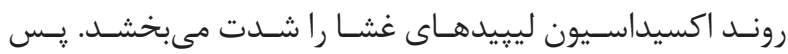

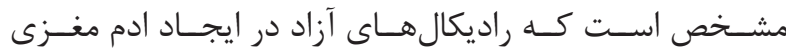

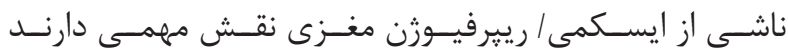

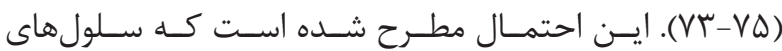

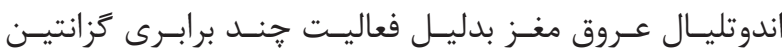

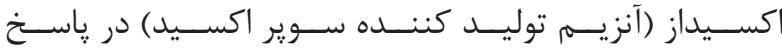

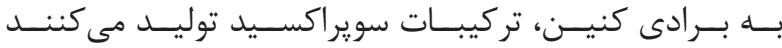
(VG، VV)

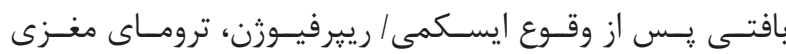

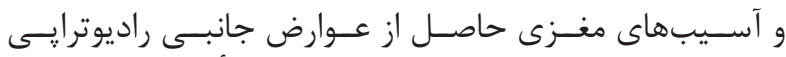

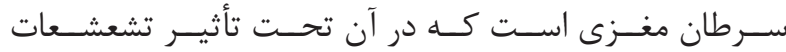

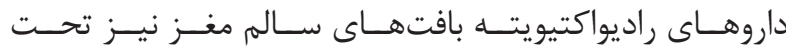

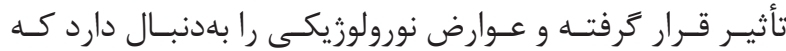

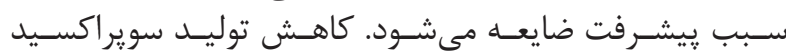

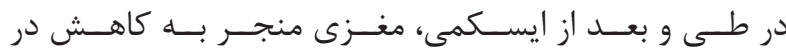

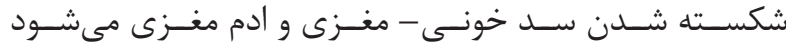

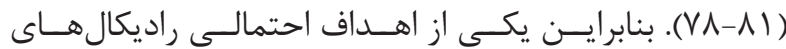

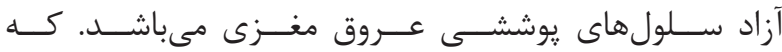

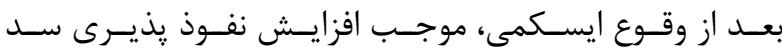

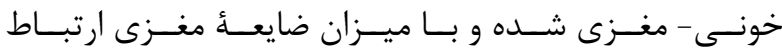
مسـتقيم دارد (

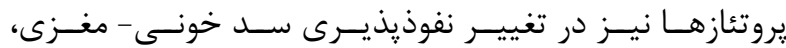

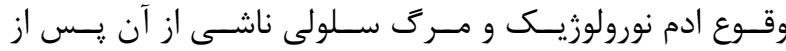

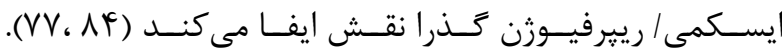

\section{اختلالات كولينرزيك ائل}

عملكردهــاى سيسـتم كولينرزيـــ در مغــز بسـيار كســـرده

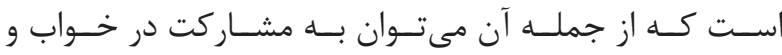

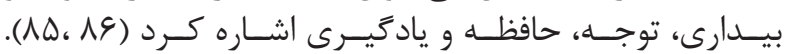

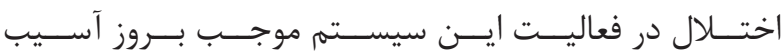

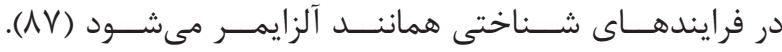

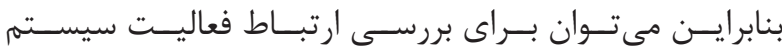

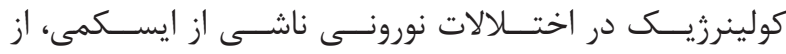

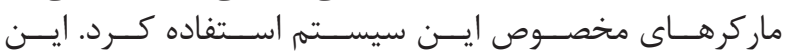

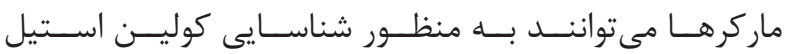

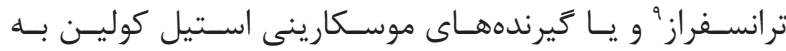

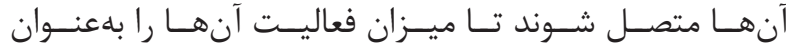

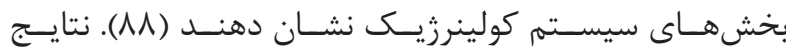

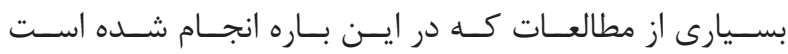

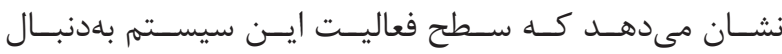

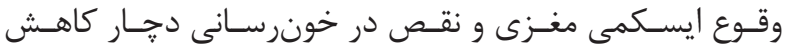

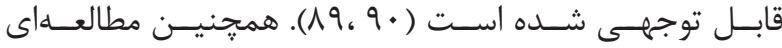

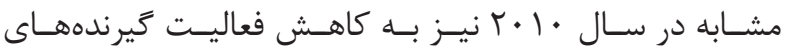

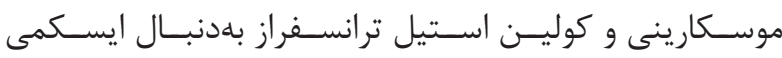

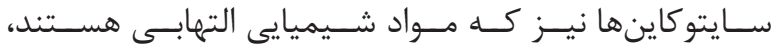

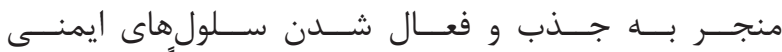

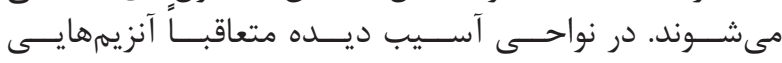

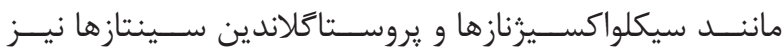

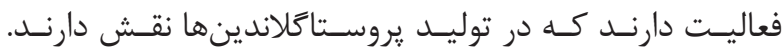

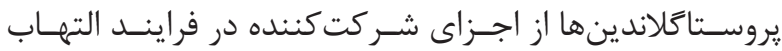

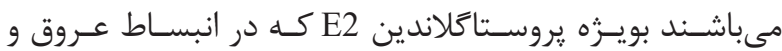

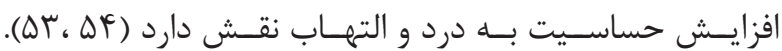

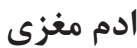

ادم مغـزى ناشـى از تجمــع آب درون سـلول يـا فضـاى بــرون

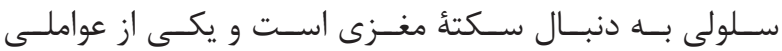

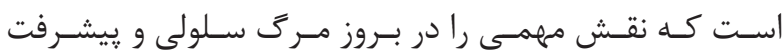

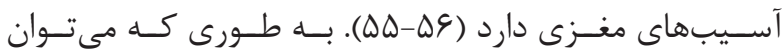

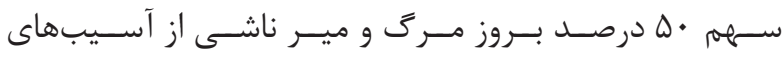

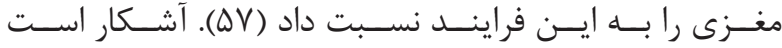

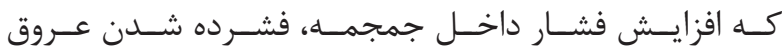

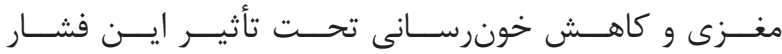

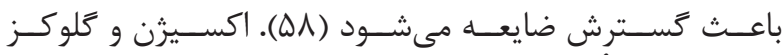

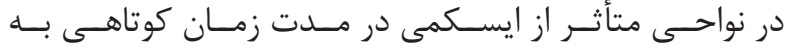

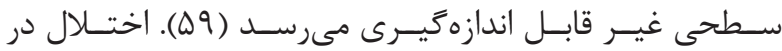

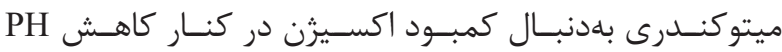

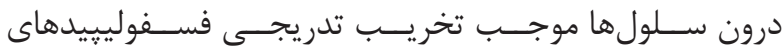

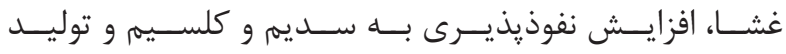

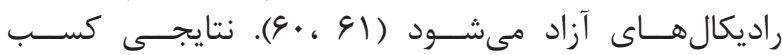

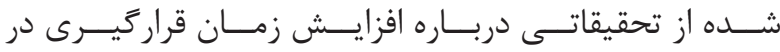

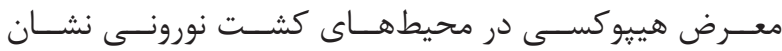

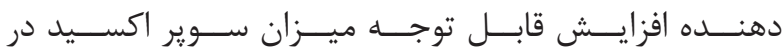

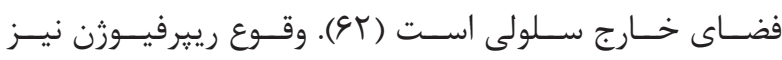

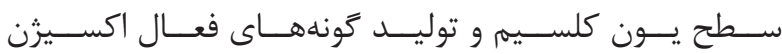

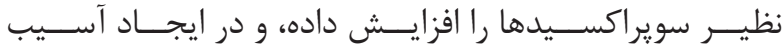

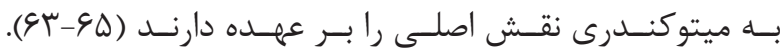

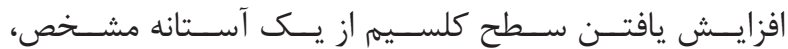

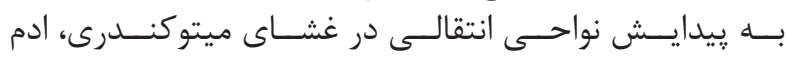

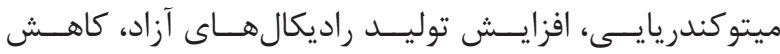

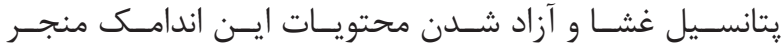

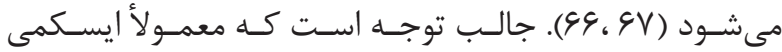

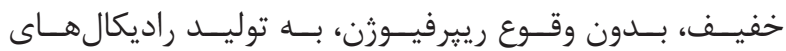

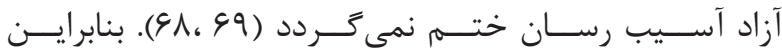

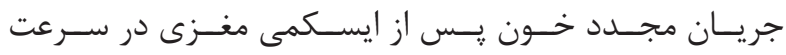

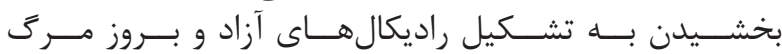

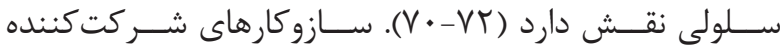

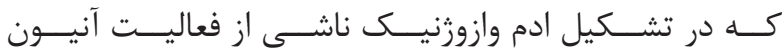

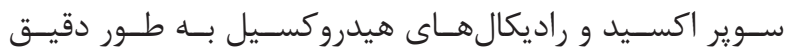

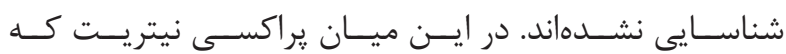




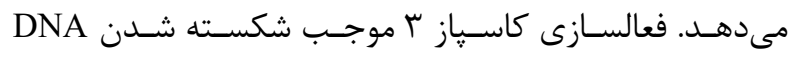

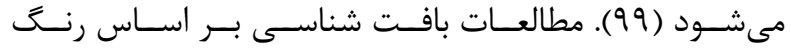

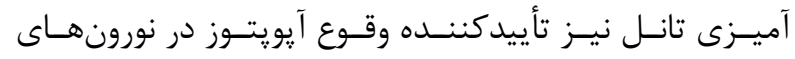

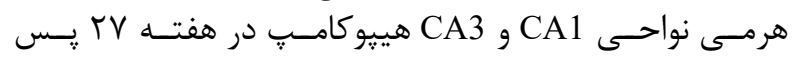

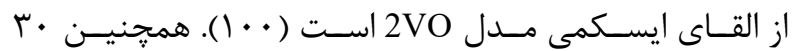

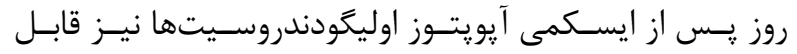

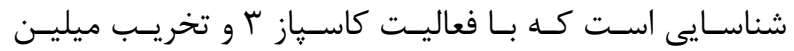

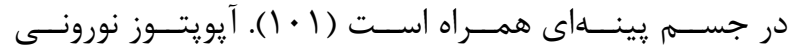

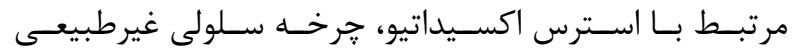

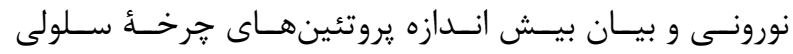

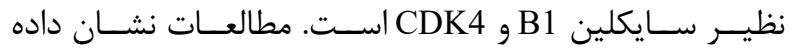

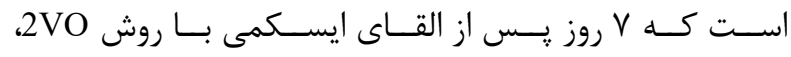

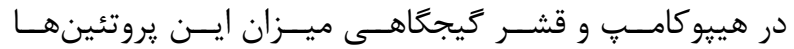

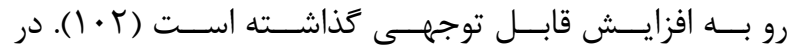

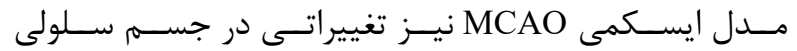

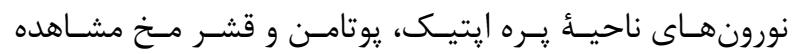

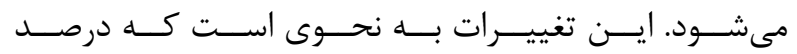

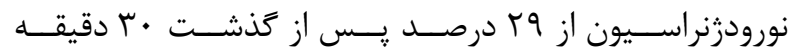

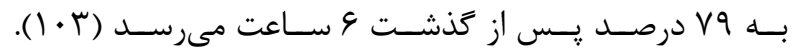
آسيب هيبيوكامب

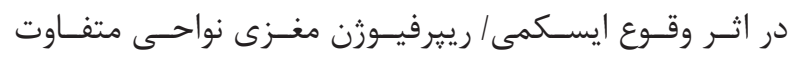

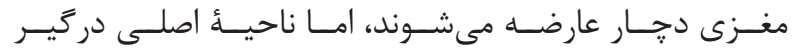

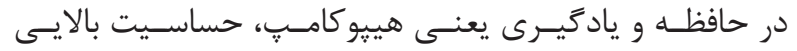

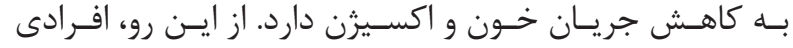

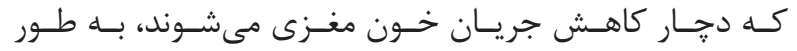

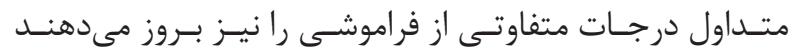

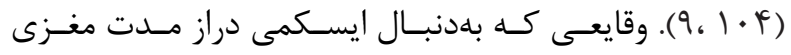

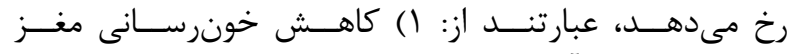

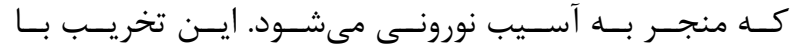

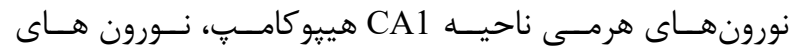

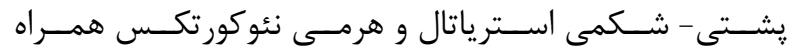

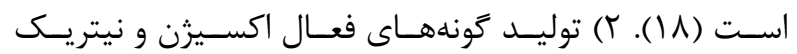

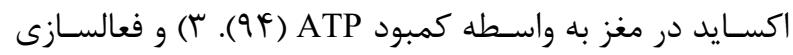

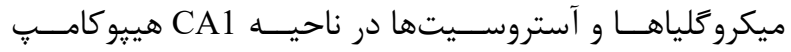

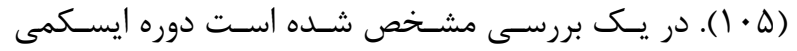

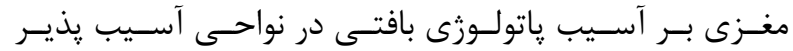

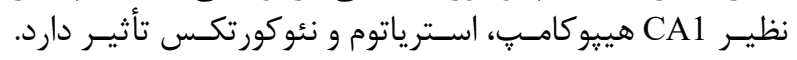

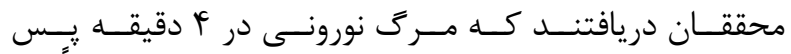

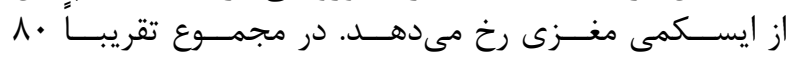

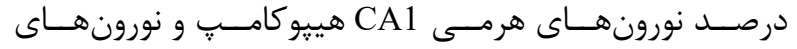

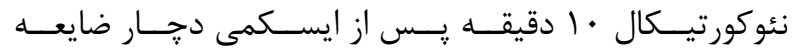

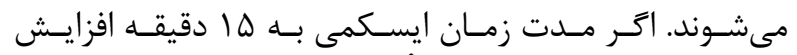

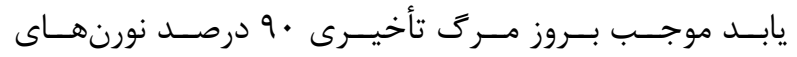

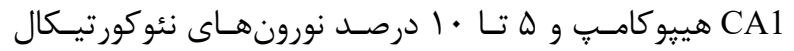

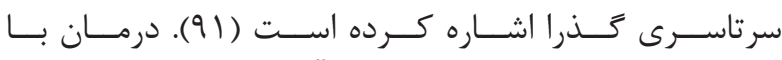

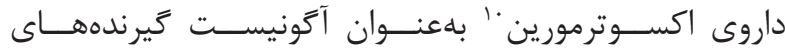

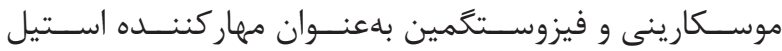

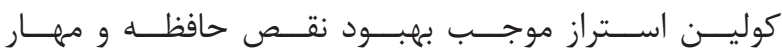

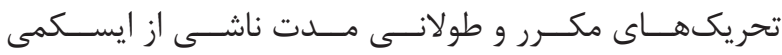

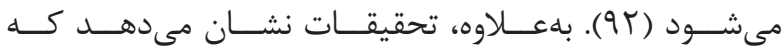

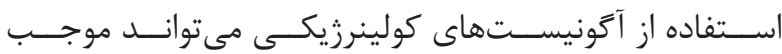

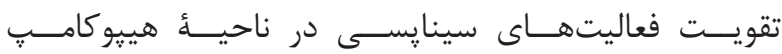

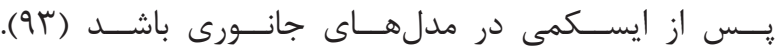

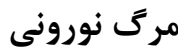

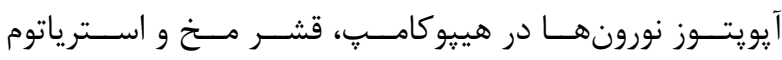

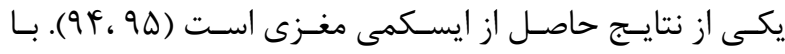

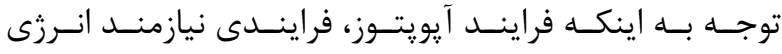

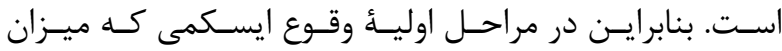

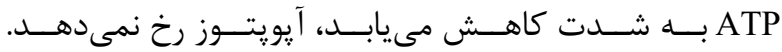

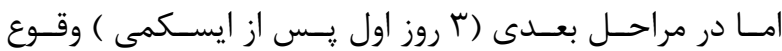

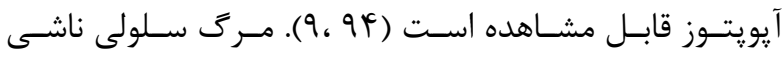

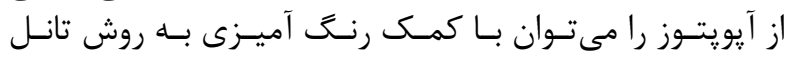

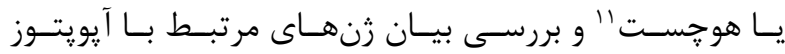

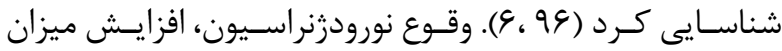

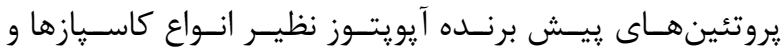

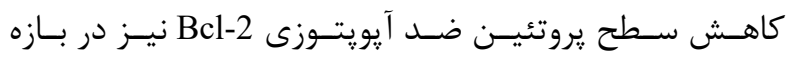

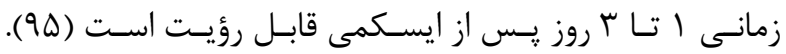

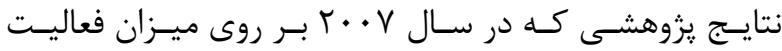

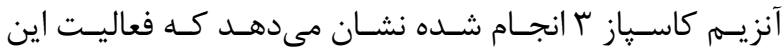

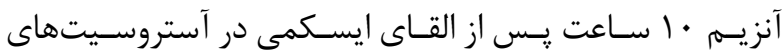

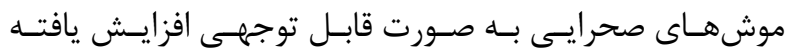

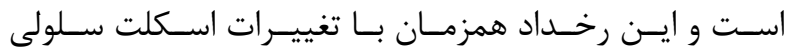

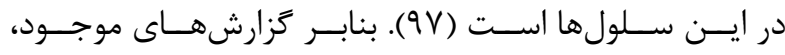

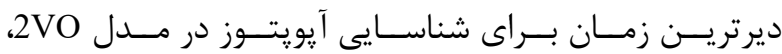

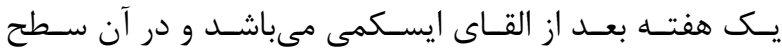

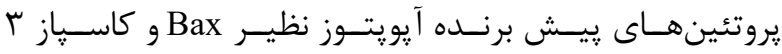

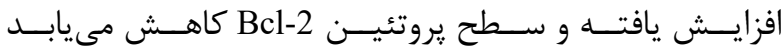

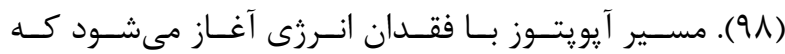

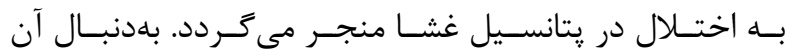

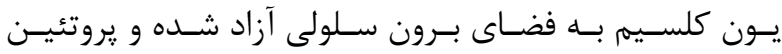

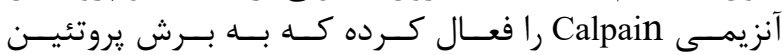
(BH3 Interacting-Domain Death (BID

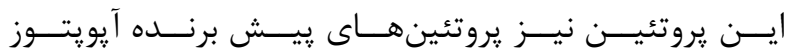

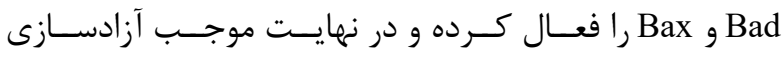

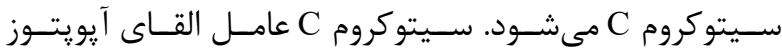

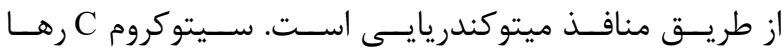

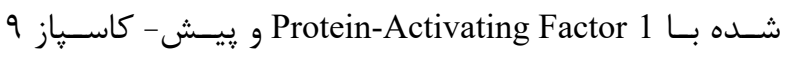

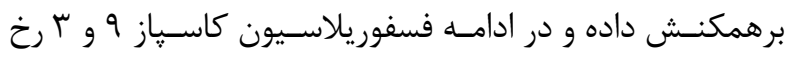




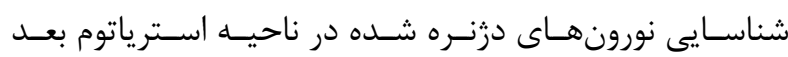

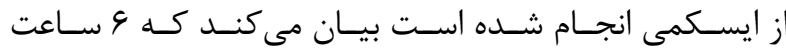

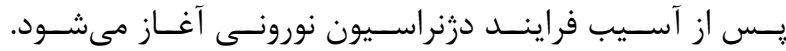

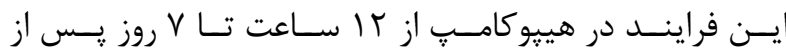

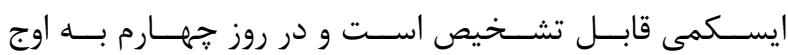

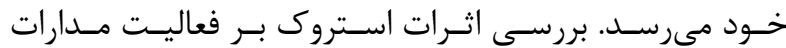

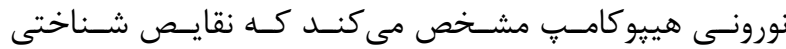

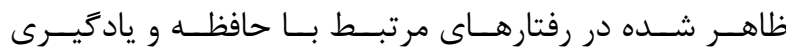

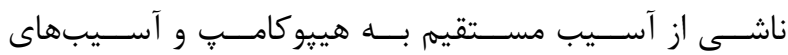

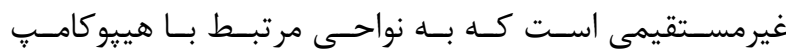

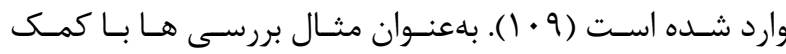

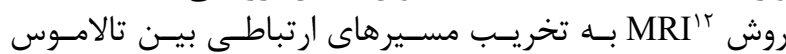

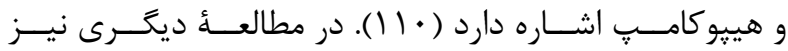

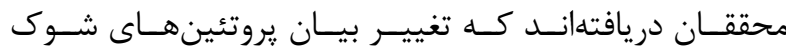

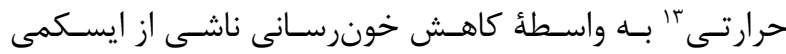

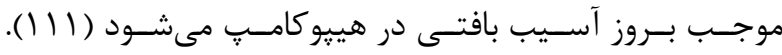

نتيجه كيرى

ايسـكمى مغــزى يكـى از حـوادث عروقـى محســوب مى شــود

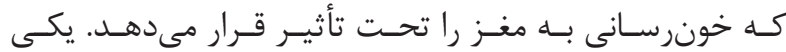

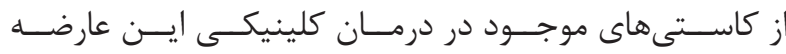

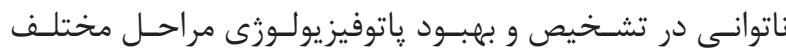

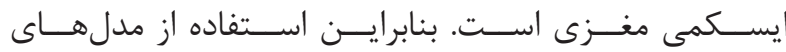

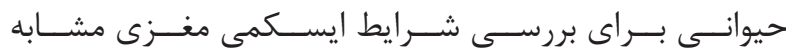

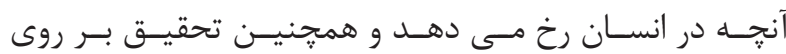

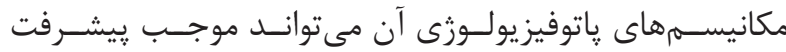

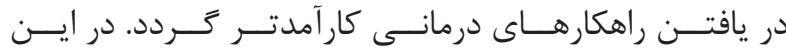

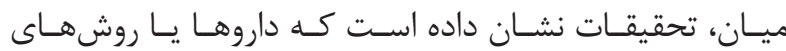

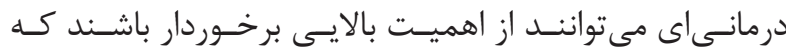

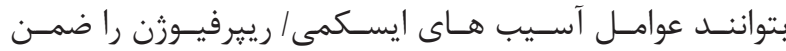

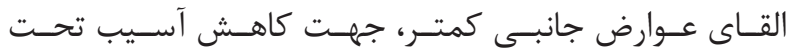

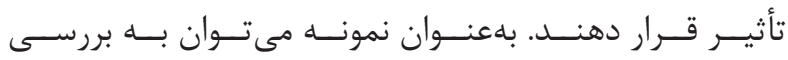

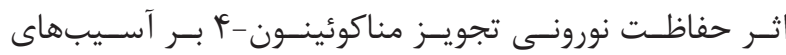

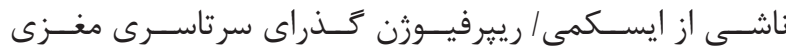

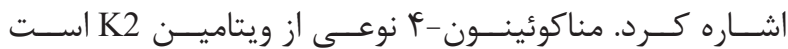

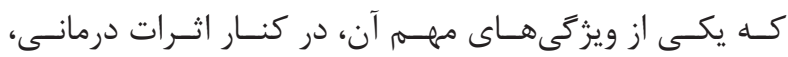

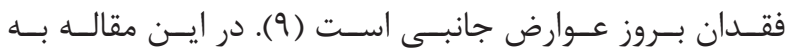

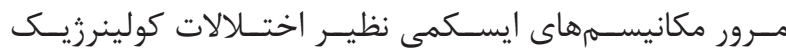

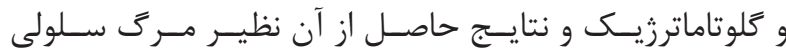

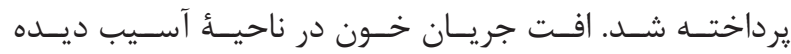

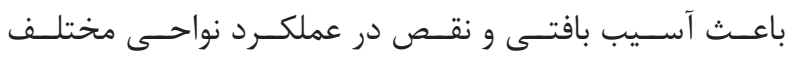

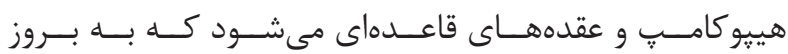

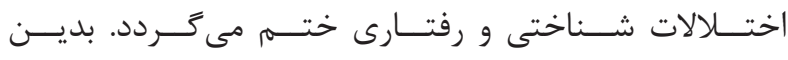

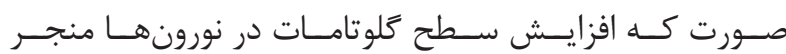

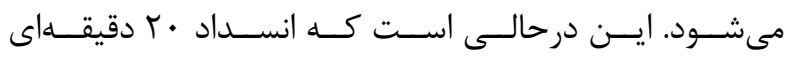

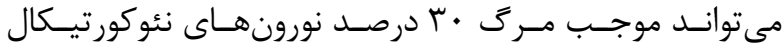

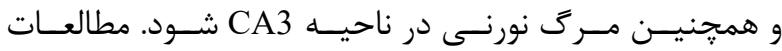

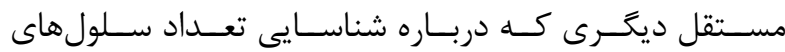

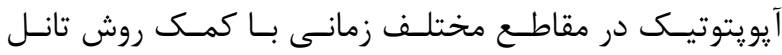

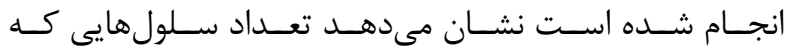

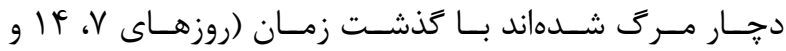

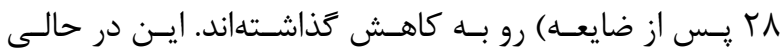

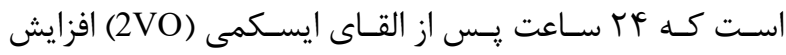

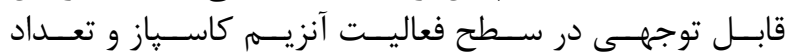

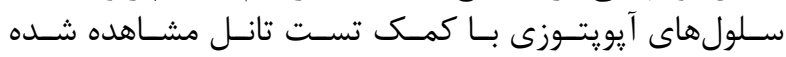

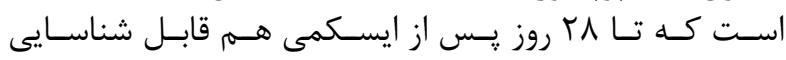

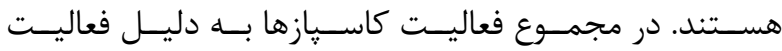

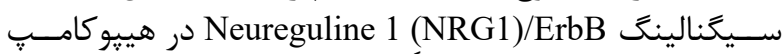

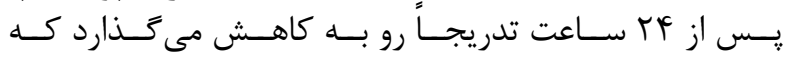

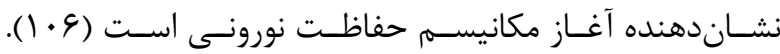

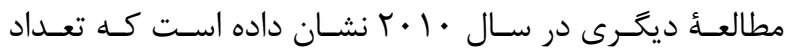

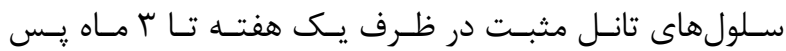

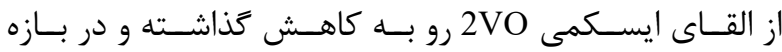

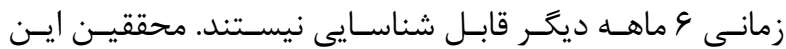

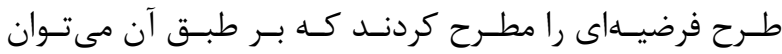

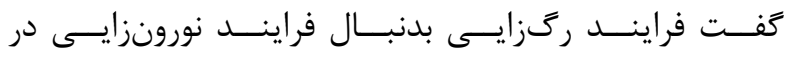

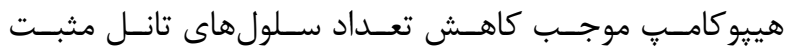

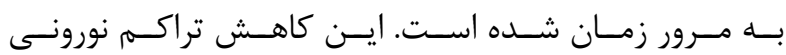

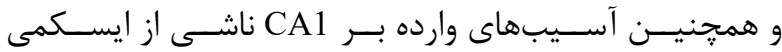

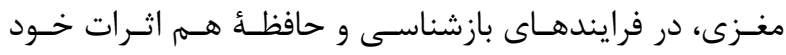

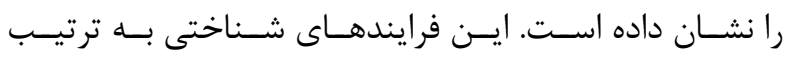

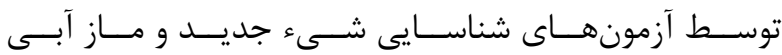

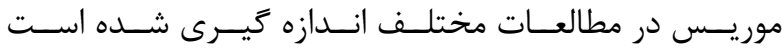

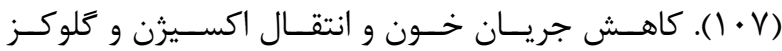

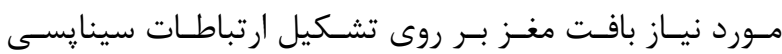

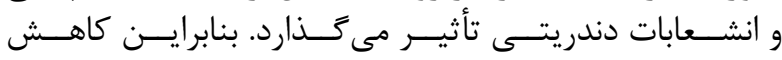

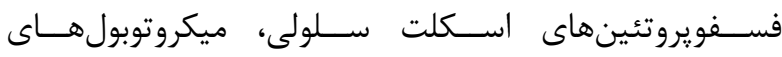

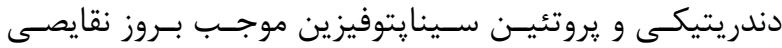

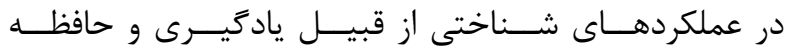

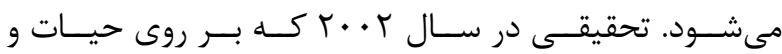

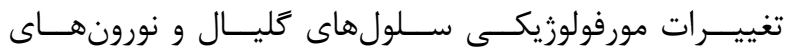

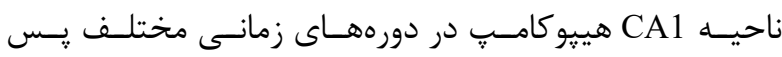

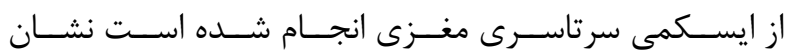

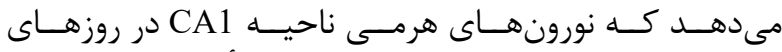

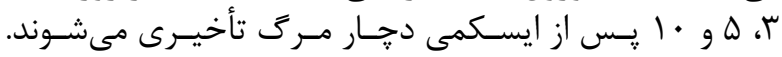

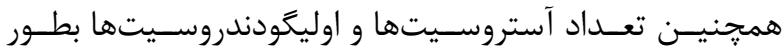

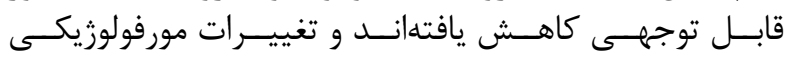

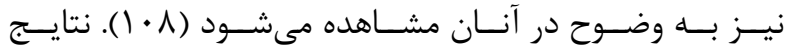

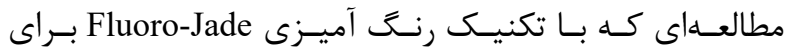




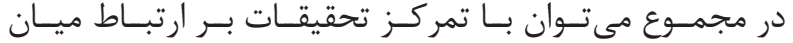

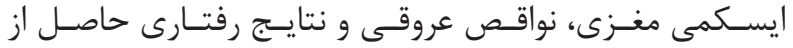

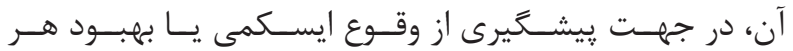

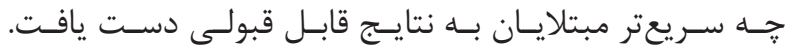

1. Bacigaluppi M, Comi G, Hermann DM. Animal models of ischemic stroke. Part two: modeling cerebral ischemia. The Open Neurology Journal. 2010; 4: 34.

2. Abdolmaleki A, Akram M, Saeed MM, Asadi A, Kajkolah M. Herbal Medicine as Neuroprotective Potential Agent in Human and Animal Models: A Historical Overview. Journal of Pharmaceutical Care. 2020; 8(2): 75-82.

3. Banerjee A. Pathology of cerebrovascular disease. Neurology India. 2000; 48(4): 305.

4. Friedman HS. Tissue plasminogen activator for acute ischemic stroke. N Engl J Med. 1995; 333(24): 1581-7.

5. Kempski O, editor Cerebral edema 2001.

6. Rosenberg GA. Ischemic brain edema. Progress in cardiovascular diseases. 1999; 42(3): 209-16.

7. Brainin $\mathrm{M}$, Dachenhausen A, Steiner M. Epidemiology of stroke]. Wiener medizinische Wochenschrift (1946). 2003; 153(1-2): 3.

8. Nagahiro S, Uno $\mathrm{M}$, Sato $\mathrm{K}$, Goto $\mathrm{S}$, Morioka M, Ushio Y. Pathophysiology and treatment of cerebral ischemia. The journal of medical investigation: JMI. 1998; 45(1-4): 57.

9. Farhadi Moghadam B, Fereidoni M. Neuroprotective effect of menaquinone-4 (MK-4) on transient global cerebral ischemia/reperfusion injury in rat. Plos one. 2020; 15(3): e0229769.

10. Traystman RJ. Animal models of focal and global cerebral ischemia. ILAR journal. 2003; 44(2): 85-95.

11. Miettinen S, Fusco FR, Yrjänheikki J, R Keinänen, Hirvonen T, Roivainen R, et al. Spreading depression and focal brain ischemia induce cyclooxygenase- 2 in cortical neurons through N-methyl-D-aspartic acidreceptors and phospholipase A2. Proceedings of the National Academy of Sciences. 1997; 94(12): 6500.

12. Khoshnazar M, Parvardeh S, Bigdeli MR. Alpha-pinene exerts neuroprotective effects via antiinflammatory and anti-apoptotic mechanisms in a rat model of focal cerebral ischemia-reperfusion. J Stroke Cerebrovasc Dis. 2020; 29(8): 104977.

13. Wang J, Ma C, Zhu J, Rao G, Li H. Effect of

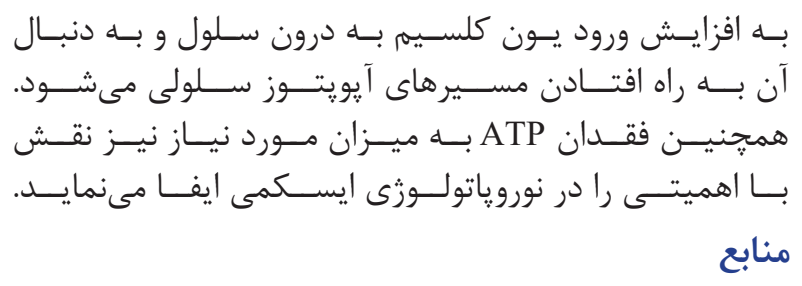

3-aminobenzamide on the ultrastructure of astrocytes and microvessels after focal cerebral ischemia in rats. Dose-Response. 2020; 18(1): 1559325819901242.

14. Kloska SP, Wintermark M, Engelhorn T, Fiebach JB. Acute stroke magnetic resonance imaging: current status and future perspective. Neuroradiology. 2010; 52(3): 189-201.

15. Astrup J, Siesjo BK, Symon L. Thresholds in cerebral ischemia-the ischemic penumbra. $\quad$ Stroke. 1981; 12(6): 723-5.

16. Elzawahry H, Hernandez-Frau P, Behrouz R, Clark M. Reperfusion injury in stroke. 2009.

17. Krause GS, Kumar K, White BC, Aust SD, Wiegenstein JG. Ischemia, resuscitation, and reperfusion: mechanisms of tissue injury and prospects for protection. American heart journal. 1986; 111(4): 768-80.

18. Damodaran T, Hassan Z, Navaratnam V, Muzaimi M, Ng G, Müller CP, et al. Time course of motor and cognitive functions after chronic cerebral ischemia in rats. Behavioural brain research. 2014; 275: 252-8.

19.Candelario-JalilE, MhaduNH,Al-DalainSM, Martínez G, León OS. Time course of oxidative damage in different brain regions following transient cerebral ischemia in gerbils. Neuroscience research. 2001; 41(3): 233-41.

20. Rothman SM, Olney JW. Glutamate and the pathophysiology of hypoxic-ischemic brain damage. Annals of neurology. 1986; 19(2): 105-11.

21. Lo EH, Moskowitz MA, Jacobs TP. Exciting, Radical, Suicidal. Stroke. 2005; 36(2): 189-92.

22. O'Collins VE, Macleod MR, Donnan GA, Horky LL, van der Worp BH, Howells DW. 1,026 experimental treatments in acute stroke. Annals of neurology. 2006; 59(3): 467-77.

23. Heiss W-D, Zeiler K, Havelec L, Reisner T, Bruck J. Long-term prognosis in stroke related to cerebral blood flow. Archives of neurology. 1977; 34(11): 671-6.

24. Meyer JS, McClintic KL, Rogers RL, Sims P, Mortel KF. Aetiological considerations and risk factors for multi-infarct dementia. Journal of Neurology, Neurosurgery \& Psychiatry. 1988; 51(12): 1489-97. 


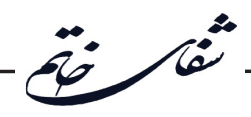

25. Sopala M, Danysz W. Chronic cerebral hypoperfusion in the rat enhances age-related deficits in spatial memory. Journal of neural transmission. 2001; 108(12): 1445-56.

26. Otori T, Katsumata T, Muramatsu H, Kashiwagi F, Katayama Y, Terashi A. Long-term measurement of cerebral blood flow and metabolism in a rat chronic hypoperfusion model. Clinical and Experimental Pharmacology and Physiology. 2003; 30(4): 266-72.

27. Coyle P, Panzenbeck MJ. Collateral development after carotid artery occlusion in Fischer 344 rats. Stroke. 1990; 21(2): 316-21.

28. Nikolova S, Moyanova S, Hughes S, BellyouCamilleri M, Lee T-Y, Bartha R. Endothelin-1 induced MCAO: dose dependency of cerebral blood flow. Journal of neuroscience methods. 2009; 179(1): 22-8.

29. Sanderson TH, Reynolds CA, Kumar R, Przyklenk $\mathrm{K}$, Hüttemann M. Molecular mechanisms of ischemiareperfusioninjuryinbrain:pivotalroleofthemitochondrial membrane potential in reactive oxygen species generation. Molecular neurobiology. 2013; 47(1): 9-23.

30. Ghayour MB, Abdolmaleki A, Behnam-Rassouli M. TheeffectofRiluzoleonfunctionalrecoveryoflocomotion in the rat sciatic nerve crush model. European Journal of Trauma and Emergency Surgery. 2017; 43(5): 691-9.

31. Siesjö BK. Pathophysiology and treatment of focal cerebral ischemia: Part I: Pathophysiology. Journal of neurosurgery. 1992; 77(2): 169-84.

32. Belov Kirdajova D, Kriska J, Tureckova J, Anderova M. Ischemia-Triggered Glutamate Excitotoxicity From the Perspective of Glial Cells. Front Cell Neurosci. 2020; 14: 51.

33. Dirnagl U, Iadecola C, Moskowitz MA. Pathobiology of ischaemic stroke: an integrated view. Trends in neurosciences. 1999; 22(9): 391-7.

34. Reiken S, Lacampagne A, ZhouH, Kherani A, Lehnart SE, Ward C, et al. PKA phosphorylation activates the calcium release channel (ryanodine receptor) in skeletal muscle. The Journal of cell biology. 2003; 160(6):919-28.

35. Lin T-N, He YY, Wu G, Khan M, Hsu CY. Effect of brain edema on infarct volume in a focal cerebral ischemia model in rats. Stroke. 1993; 24(1): 117-21.

36. Hossmann K. Periinfarct depolarizations. Cerebrovascular and brain metabolism reviews. $\quad$ 1995; $8(3)$ 195-208.

37. Kemmerling U, Muñoz P, Müller M, Sánchez G,
Aylwin ML, Klann E, et al. Calcium release by ryanodine receptors mediates hydrogen peroxide-induced activation of ERK and CREB phosphorylation in N2a cells and hippocampalneurons. Cell calcium. 2007;41(5):491-502.

38. Granados MP, Salido GM, González A, Pariente JA. Dose-dependent effect of hydrogen peroxide on calcium mobilization in mouse pancreatic acinar cells. Biochemistry and cell biology. 2006; 84(1): 39-48.

39. Li J, Wang H, Rosenberg PA. Vitamin K prevents oxidative cell death by inhibiting activation of 12lipoxygenase in developing oligodendrocytes. Journal of neuroscience research. 2009; 87(9): 1997-2005.

40. Chamorro Á, Dirnagl U, Urra X, Planas AM. Neuroprotection in acute stroke: targeting excitotoxicity, oxidative and nitrosative stress, and inflammation. The Lancet Neurology. 2016; 15(8): 869-81.

41. Powers SK, Jackson MJ. Exercise-induced oxidative stress: cellular mechanisms and impact on muscle force production. Physiological reviews. 2008; 88(4): 1243-76.

42. Roy P, Roy SK, Mitra A, Kulkarni AP. Superoxide generation by lipoxygenase in the presence of NADH and NADPH. Biochimica et Biophysica Acta (BBA)Lipids and Lipid Metabolism. 1994; 1214(2): 171-9.

43. Zuo L, Christofi FL, Wright VP, Bao S, Clanton TL. Lipoxygenase-dependent superoxide release in skeletal muscle.Journalof AppliedPhysiology.2004;97(2):661-8.

44. Abdolmaleki A, Fereidoni M, Asgari A. Analgesic and Anti-Inflammatory Effects of Hydroalcoholic Extract of Salvia multicaulis on Male Rats. Sciences. 2015; 21(2): 121-8.

45. Pradillo JM, Murray KN, Coutts GA, Moraga A, Oroz-Gonjar F, Boutin H, et al. Reparative effects of interleukin-1 receptor antagonist in young and aged/co-morbid rodents after cerebral ischemia. Brain, Behavior, and Immunity. 2017; 61: 117-26.

46. Honardoost M, Soleimanjahi H, Rajaei F. Apoptosis: programmed cell death. JQUMS. 2013; 17(3): 48-57.

47. Wallach D, Kang T-B, Kovalenko A. Concepts of tissue injury and cell death in inflammation: a historical perspective. Nature Reviews Immunology. 2014; 14(1): 51-9.

48. Chen L, Deng H, Cui H, Fang J, Zuo Z, Deng J, et al. Inflammatory responses and inflammation-associated diseases in organs. Oncotarget. 2018; 9(6): 7204.

49. Svíženská I, Dubový P, Šulcová A. Cannabinoid 
receptors 1 and 2 ( $\mathrm{CB} 1$ and $\mathrm{CB} 2)$, their distribution, ligands and functional involvement in nervous system structures-a short review. Pharmacology Biochemistry and Behavior. 2008; 90(4): 501-11.

50. Hajipoor F, Fereidoni M, Moghimi A. Effects of Intrathecal Administration of Vitamin K2 on Pain in the Tail Flick and Formalin Test in Rats. Journal of Mazandaran University of Medical Sciences. 2014; 24(119): 132-40.

51. Gröger M, Lebesgue D, Pruneau D, Relton J, Kim S-W, Nussberger J, et al. Release of bradykinin and expression of kinin B2 receptors in the brain: role for cell death and brain edema formation after focal cerebral ischemia in mice. Journal of Cerebral Blood Flow \& Metabolism. 2005; 25(8): 978-89.

52. Lehmberg J, Beck J, Baethmann A, Uhl E. Influence of the Bradykinin B1/B2-ReceptorAntagonist B 9430 on the Cerebral Microcirculation and Outcome of Gerbils from Global Cerebral Ischemia. Brain Edema XI: Springer; 2000. p. 39-41.

53. Kandel ER, Schwartz JH, Jessell TM, Siegelbaum SA, Hudspeth AJ. Principles of neural science: McGraw-hill New York; 2000.

54. Williams CS, Mann M, DuBois RN. The role of cyclooxygenases in inflammation, cancer, and development. Oncogene. 1999; 18(55).

55. Cellular OR. The pathophysiology of brain edema and elevated intracranial pressure. Cleveland Clinic journal of medicine. 2004; 71: S7.

56. Sharma HS, Westman J, Nyberg F. Pathophysiology of brain edema and cell changes following hyperthermic brain injury. Progress in brain research. 1998; 115: 351-412.

57. Bennett MVL, Contreras JE, Bukauskas FF, Sez JC. New roles for astrocytes: gap junction hemichannels have something to communicate. Trends in Neurosciences. 2003; 26(11): 610-7.

58. Dietrich WD. Neurobiology of stroke. International review of neurobiology. 1998; 42: 55-101.

59. Stout CE, Costantin JL, Naus CCG, Charles AC. Intercellular calcium signaling in astrocytes via ATP release through connexin hemichannels. Journal of Biological Chemistry. 2002; 277(12): 10482-8.

60. Hormuzdi SG, Pais I, LeBeau FEN, Towers SK, Rozov A, Buhl EH, et al. Impaired electrical signaling disrupts gamma frequency oscillations in connexin 36-deficient mice. Neuron. 2001; 31(3): 487-95.
61. Abdolmaleki A, Zahri S, Bayrami A. Rosuvastatin enhanced functional recovery after sciatic nerve injury in the rat. European Journal of Pharmacology. 2020: 173260.

62. Scemes E, Dermietzel R, Spray DC. Calcium waves between astrocytes from Cx43 knockout mice. Glia. 1998; 24(1) :65.

63. Micevych PE, Abelson L. Distribution of mRNAs coding for liver and heart gap junction proteins in the rat central nervous system. The Journal of comparative neurology. 1991; 305(1): 96-118.

64. Belliveau DJ, Naus CGC. Cellular Localization of Gap Junction mRNAs in Developing Rat Brain (Part 2 of 2). Developmental neuroscience. 1995; 17(2): 90-6.

65. Spray DC, Dermietzel R. X-linked dominant Charcot Marie Tooth disease and other potential gap-junction diseases of the nervous system. Trends in Neurosciences. 1995; 18(6): 256-62.

66. Suter U, Snipes GJ. Biology and genetics of hereditary motor and sensory neuropathies. Annual review of neuroscience. 1995; 18(1): 45-75.

67. Giaume C, Venance L. Gap junctions in brain glial cells and development. Perspectives on developmental neurobiology. 1995; 2(4): 335.

68. Cino M, Del Maestro RF. Generation of hydrogen peroxidebybrainmitochondria: theeffectofreoxygenation following postdecapitative ischemia. Archives of biochemistry and biophysics. 1989; 269(2): 623 .

69. Love S. Oxidative stress in brain ischemia. Brain Pathology. 1999; 9(1): 119-31.

70. Piantadosi CA, Zhang J. Mitochondrial generation of reactive oxygen species after brain ischemia in the rat. Stroke. 1996; 27(2): 327-32.

71. Chan PH. Reactive oxygen radicals in signaling and damage in the ischemic brain. Journal of Cerebral Blood Flow \& Metabolism. 2001; 21(1): 2-14.

72. Liu PK, Hsu CY, Dizdaroglu M, Floyd RA, Kow YW, Karakaya A, et al. Damage, repair, and mutagenesis in nuclear genes after mouse forebrain ischemia reperfusion. The Journal of neuroscience. 1996; 16(21): 6795-806.

73. Ikeda Y, Long DM. The molecular basis of brain injury and brain edema: the role of oxygen free radicals. Neurosurgery. 1990; 27(1): 1.

74. Chan PH, Yurko M, Fishman RA. Phospholipid degradation and cellular edema induced by free radicals in brain cortical slices. Journal 
of neurochemistry.

1982; 38(2): $\quad 525-31$.

75. Heo JH, Han SW, Lee SK. Free radicals as triggers of brain edema formation after stroke. Free Radical Biology and Medicine. 2005; 39(1): 51-70.

76. Stanimirovic D, Satoh K. Inflammatory mediators of cerebral endothelium: a role in ischemic brain inflammation. Brain Pathology. 2000; 10(1): 113-26.

77. Abbott NJ. Inflammatory mediators and modulation of blood brain barrier permeability. Cellular and molecular neurobiology. 2000; 20(2): 131-47.

78. Beaumont A, Marmarou A, Hayasaki K, Barzo P, Fatouros P, Corwin F, et al. The permissive nature of blood brain barrier (BBB) opening in edema formation following traumatic brain injury. Acta Neurochirurgica. 2001: 125-9.

79. d'Avella D, Cicciarello R, Angileri FF, Lucerna S, La Torre D, Tomasello F. Radiation-induced blood-brain barrier changes: pathophysiological mechanisms and clinical implications. ACTA NEUROCHIRURGICA SUPPLEMENTUM-WIEN-. $1998 ; \quad 71$ : 282-4.

80. Nordal RA, Wong CS. Molecular targets in radiation-induced blood-brain barrier disruption. International Journal of Radiation Oncology* Biology* Physics. 2005; 62(1): 279-87.

81. Yang Z, Bai S, Gu B, Peng S, Liao W, Liu J. Radiation-induced brain injury after radiotherapy for brain tumor. Molecular Considerations and Evolving Surgical Management Issues in the Treatment of Patients with a Brain Tumor. 2015.

82. Betz AL, Keep RF, Beer ME, Ren X. Blood-brain barrier permeability and brain concentration of sodium, potassium, and chloride during focal ischemia. Journal of Cerebral Blood Flow \& Metabolism. 1994; 14(1): 29-37.

83. Hatashita S, Hoff JT. Brain edema and cerebrovascular permeability during cerebral ischemia in rats. Stroke. 1990; 21(4): 582-8.

84. Yang GY, Betz AL. Reperfusion-induced injury to the blood-brain barrier after middle cerebral artery occlusion in rats. Stroke. 1994; 25(8): 1658-64.

85. Thiel CM. Cholinergic modulation of learning and memory in the human brain as detected with functional neuroimaging. Neurobiology of learning and memory. 2003; 80(3): 234-44.

86. Hampel H, Mesulam MM, Cuello AC, Farlow MR, Giacobini E, Grossberg GT, et al. The cholinergic system in the pathophysiology and treatment of
Alzheimer's disease. Brain. 2018; 141(7): 1917-33.

87. de Souza Silva MA, Lenz B, Rotter A, Biermann T, Peters O, Ramirez A, et al. Neurokinin3 receptor as a target to predict and improve learning and memory in the aged organism. Proceedings of the National Academy of Sciences. 2013; 110(37): 15097-102.

88. Tanaka K-i, Ogawa N, Asanuma M, Kondo Y, Nomura M. Relationship between cholinergic dysfunction and discrimination learning disabilities in Wistar rats following chronic cerebral hypoperfusion. Brain research. 1996; 729(1): 55-65.

89. Ahmad A, Khan MM, Javed H, Raza SS, Ishrat T, Khan MB, et al. Edaravone ameliorates oxidative stress associated cholinergic dysfunction and limits apoptotic responsefollowing focalcerebralischemiainrat. Molecular and cellular biochemistry. 2012; 367(1-2): 215-25.

90. Ishimaru H, Takahashi A, Ikarashi Y, Maruyama Y. Pentobarbital protects against CA1 pyramidal cell death but not dysfunction of hippocampal cholinergic neurons following transient ischemia. Brain research. 1995; 673(1): 112-8.

91. Zakharova E, Storozheva Z, Dudchenko A, Kubatiev A. Chronic cerebral ischaemia forms new cholinergic mechanisms of learning and memory. International Journal of Alzheimer's Disease. 2010; 2010.

92. Damodaran T, Müller CP, Hassan Z. Chronic cerebral hypoperfusion-induced memory impairment and hippocampal long-term potentiation deficits are improved by cholinergic stimulation in rats. Pharmacological Reports. 2019; 71(3): 443-8.

93. Azam NF, Stanyard RA, Mat NH, Hassan Z. Cholinergic modulation of hippocampal long-term potentiation in chronic cerebral hypoperfused rats. Neuroscience Research Notes. 2018; 1(1): 42-57.

94. Farkas E, Luiten PG, Bari F. Permanent, bilateral common carotid artery occlusion in the rat: a model for chroniccerebralhypoperfusion-relatedneurodegenerative diseases. Brain research reviews. 2007; 54(1): 162-80.

95. Stanojlović M, Guševac I, Grković I, Zlatković J, Mitrović N, Zarić M, et al. Effects of chronic cerebral hypoperfusion and low-dose progesterone treatment on apoptotic processes, expression and subcellular localization of key elements within Akt and Erk signaling pathways in rat hippocampus. Neuroscience. 2015; 311: 308-21.

96. Wang J-K, Guo Q, Zhang X-W, Wang L-C, Liu Q, Tu P-F, et al. Aglaia odorata Lour. extract inhibit ischemic neuronal injury potentially via suppressing 
p53/Puma-mediated mitochondrial apoptosis pathway. Journal of Ethnopharmacology. 2020; 248: 112336.

97. Acarin L, Villapol S, Faiz M, Rohn TT, Castellano B, González B. Caspase-3 activation in astrocytes following postnatal excitotoxic damage correlates with cytoskeletal remodeling but not with cell death or proliferation. Glia. 2007; 55(9): 954-65.

98. Liu G, Wang $\mathrm{T}$, Wang $\mathrm{T}$, Song J, Zhou Z. Effects of apoptosis related proteins caspase 3, $\mathrm{Bax}$ and $\mathrm{Bcl} 2$ on cerebral ischemia rats. Biomedical reports. 2013; 1(6): 861-7.

99. Broughton BR, Reutens DC, Sobey CG. Apoptotic mechanisms after cerebral ischemia. Stroke. 2009; 40(5): e331-e9.

100. Bennett SA, Tenniswood M, Chen J-H, Davidson CM, Keyes MT, Fortin T, et al. Chronic cerebral hypoperfusion elicits neuronal apoptosis and behavioral impairment. Neuroreport. 1998; 9(1): 161-6.

101.CaiQ,YaoZ,LiH.Catalpolpromotesoligodendrocyte survival and oligodendrocyte progenitor differentiation via the Akt signaling pathway in rats with chronic cerebral hypoperfusion. Brain research. 2014; 1560: 27-35.

102. Xu L, Di Q, Zhang Y. Cell cycle proteins preceded neuronal death after chronic cerebral hypoperfusion in rats. Neurological research. 2008; 30(9): 932-9.

103. Iadecola C, Zhang F, Xu S, Casey R, Ross ME. Inducible nitric oxide synthase gene expression in brain following cerebral ischemia. Journal of Cerebral Blood Flow \& Metabolism. 1995; 15(3): 378-84.

104. Kho AR, Choi BY, Lee SH, Hong DK, Lee $\mathrm{SH}$, Jeong JH, et al. Effects of protocatechuic acid (PCA) on global cerebral ischemia-induced hippocampal neuronal death. International journal of molecular sciences. 2018; 19(5): 1420

105. Farkas E, Donka G, de Vos RA, Mihály A, Bari F, Luiten PG. Experimental cerebral hypoperfusion induces white matter injury and microglial activation in the rat brain. Acta Neuropathologica. 2004; 108(1): 57-64.

106. Hei Y, Chen R, Yi X, Wei L, Long Q, Liu W. The expression of hippocampal NRG1/ErbB4 correlates with neuronal apoptosis, but not with glial activation during chronic cerebral hypoperfusion. Frontiers in aging neuroscience. 2018; 10: 149.

107. De Jong G, Farkas E, Stienstra C, Plass J, Keijser J, De la Torre J, et al. Cerebral hypoperfusion yields capillary damage in the hippocampal CA1 area that correlates with spatial memory impairment. Neuroscience. 1999; 91(1): 203-10.

108. Sugawara T, Noshita N, Lewen A, Gasche Y, Ferrand-Drake M, Fujimura M, et al. Overexpression of copper/zinc superoxide dismutase in transgenic rats protects vulnerable neurons against ischemic damage by blocking the mitochondrial pathway of caspase activation. Journal of Neuroscience. 2002; 22(1): 209-17.

109. Butler TL, Kassed CA, Sanberg PR, Willing AE, Pennypacker KR. Neurodegeneration in the rat hippocampus and striatum after middle cerebral artery occlusion. Brain research. 2002; 929(2): 252-60.

110. Baumgartner P, El Amki M, Bracko O, Luft AR, Wegener S. Sensorimotor stroke alters hippocampo-thalamic network activity. Scientific reports. 2018; 8(1): 1-11.

111. Shah FA, Zeb A, Li S, Al Kury LT. Pathological Comparisons of the Hippocampal Changes in the TransientandPermeantMiddleCerebral Artery Occlusion Rat Models. Frontiers in Neurology. 2019; 10: 1178. 